\title{
A Fuzzy Logic Model for the Analysis of Ultrasonic Vibration Assisted Turning and Conventional Turning of Ti-Based Alloy
}

\author{
Riaz Muhammad (D) \\ Mechanical Engineering Department, College of Engineering, University of Bahrain, \\ Isa Town P.O. Box 32038, Bahrain; rmuhammad@uob.edu.bh
}

check for

updates

Citation: Muhammad, R. A Fuzzy Logic Model for the Analysis of Ultrasonic Vibration Assisted Turning and Conventional Turning of Ti-Based Alloy. Materials 2021, 14, 6572. https://doi.org/10.3390/ ma14216572

Academic Editor: Weronika Kruszelnicka

Received: 19 August 2021

Accepted: 13 October 2021

Published: 1 November 2021

Publisher's Note: MDPI stays neutral with regard to jurisdictional claims in published maps and institutional affiliations.

\begin{abstract}
Titanium and its alloys are largely used in various applications due its prominent mechanical properties. However, the machining of titanium alloys is associated with assured challenges, including high-strength, low thermal conductivity, and long chips produced in conventional machining processes, which result in its poor machinability. Advanced and new machining techniques have been used to improve the machinability of these alloys. Ultrasonic vibration assisted turning (UVAT) is one of these progressive machining techniques, where vibrations are imposed on the cutting insert, and this process has shown considerable improvement in terms of the machinability of hard-to-cut alloys. Therefore, selecting the right cutting parameters for conventional and assisted machining processes is critical for obtaining the anticipated dimensional accuracy and improved surface roughness of Ti-alloys. Hence, fuzzy-based algorithms were developed for the ultrasonic vibration assisted turning (UVAT) and conventional turning (CT) of the Ti-6Al7Zr3Nb4Mo0.9Nd alloy to predict the maximum process zone temperature, cutting forces, surface roughness, shear angle, and chip compression ratio for the selected range of input parameters (speed and depth-of-cut). The fuzzy-measured values were found to be in good agreement with the experimental values, indicating that the created models can be utilized to accurately predict the studied machining output parameters in CT and UVAT processes. The studied alloy resulted in discontinued chips in both the CT and UVAT processes. The achieved results also demonstrated a significant decline in the cutting forces and improvements in the surface quality in the UVAT process. Furthermore, the chip discontinuity is enhanced by the UVAT process due to the higher process zone temperature and the micro-impact imposed by the cutting tool on the workpiece.
\end{abstract}

Keywords: fuzzy logic; turning; Ti-alloys; chip formation

\section{Introduction}

The balanced set of mechanical properties, lightweight, and corrosion to resistivity of titanium alloys make it an excellent choice for bellicose environment applications [1]. However, the high strength and low thermal conductivity causes severe challenges for mechanists in industries, resulting in poor surface quality, a high rejection rate, and tool wear [2]. Additionally, the spring back effect and continuous chip production of titanium alloys results time loss in production systems.

In the past, experiments and simulations have been used to study and improve the machinability of hard-to-cut alloys. These improvements were achieved by enhancing the machining capabilities [3-6], hybrid machining techniques [7], cryogenic coolant application [8], minimum lubricant quantity [9], and alloy modification without compromising the mechanical properties of the alloy [10]. The theory of ultrasonic machining has been successfully applied to face milling and drilling operations, and significant improvements in terms of the surface quality of the finished product has been achieved [11-13].

With these advancements in simulation tools, various studies have been conducted on the machining of titanium alloys [14]. Simulation tools allow readers to obtain the required results without spending time on costly and time-consuming experimental techniques. 
Many two- and three-dimensional finite element (FE) models have been developed for the orthogonal turning of Ti alloys to examine the outcomes of materials at several machining input parameters [15-18]. Similarly, with advances in computational facilities and software, three-dimensional FE simulation models have been established for conventional and assisted oblique turning processes $[7,19]$.

With Industrial Revolution 4.0 and the advancement in CNC machines, most industries are moving toward the uninterrupted automatic machining of high-quality products. The long continuous chips produced in Ti alloys interrupt the machining time due to their entanglement with either the workpiece or cutting tool, affecting the surface quality of the finished product and the sharpness of the cutting tool, respectively. Therefore, artificial intelligence (AI) methods are becoming more desirable for the modeling of machining processes. Recently, a fuzzy-logic technique based on the combination of multivalued logic and the theory of probability to model complex engineering problems is gaining popularity in the research community [20]. Fuzzy-logic offers additional good judgment and concrete means to overcome the problem of commanding reasoning abilities confined by rules [21]. This method has been used by several investigators for the prediction of machining results based on the various input machining parameters as listed in Table 1.

Table 1. The contribution of researchers using fuzzy logic tools in machining processes in recent years.

\begin{tabular}{|c|c|c|c|c|}
\hline Process & Workpiece & Machining Input Parameter & Investigation & Reference \\
\hline DP & Al-5083 & V, FR & SQ, hole size & [20] \\
\hline EDM & SS-304 & pulse-on/off times and current & MRR and EWR & {$[22]$} \\
\hline LM & PMMA & $\begin{array}{l}\mathrm{V} \text {, power of laser, } \mathrm{CO}_{2} \text { pressure, and } \\
\text { stand-off distance }\end{array}$ & Width-kerf & [23] \\
\hline MP & Al-6061 T6 & V, DoC, and FR & SQ & {$[24]$} \\
\hline $\mathrm{TP}$ & AISI 1045 & V, DoC, and FR and approach angle & SQ, TW and, MRR & {$[25]$} \\
\hline MP & Al-6061 T6 & $\begin{array}{l}\text { Nozzle pressure, nozzle angle, and } \\
\text { nanoparticle concentration }\end{array}$ & $\begin{array}{l}\text { Cutting forces, cutting } \\
\text { temperature, and SQ }\end{array}$ & [26] \\
\hline $\mathrm{TP}$ & $\begin{array}{l}\text { Al-Si-Cu-Fe and doped } \\
\text { alloys }\end{array}$ & FR, V, and alloy type & 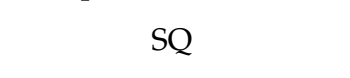 & [27] \\
\hline AWJM & Brittle materials & $\begin{array}{c}\text { Nozzle diameter, pressure of liquid, } \\
\text { mass flow rate of abrasive jet traverse } \\
\text { rate }\end{array}$ & DoC & [28] \\
\hline $\mathrm{TP}$ & Ti64 & V, DoC, FR, & $\begin{array}{l}\text { SQ, TW, and specific } \\
\text { cutting pressure }\end{array}$ & [29] \\
\hline EDM drilling & AISI-1010 Steel & $\begin{array}{l}\text { Voltage gap, spark ratio deviation, and } \\
\text { change in the deviation in spark-ratio }\end{array}$ & $\begin{array}{l}\text { Current, voltage, and } \\
\text { ignition delay time }\end{array}$ & {$[30]$} \\
\hline Wire EDM & Die steel & Thickness, FR, and corner angle & $\begin{array}{c}\text { Machining accuracy at } \\
\text { corner parts }\end{array}$ & {$[31]$} \\
\hline EDM & Die steel & $\begin{array}{l}\text { Discharge current, the polarity of the } \\
\text { workpiece, voltage at open discharge, } \\
\text { pulse-on time, duty factor, }\end{array}$ & $\begin{array}{l}\text { MRR and electrode } \\
\text { wear ratio }\end{array}$ & {$[32]$} \\
\hline EDM & Tool steel & $\begin{array}{l}\text { Powder concentration, open-circuit } \\
\text { voltage, duty cycle, pulsed duration, } \\
\text { pulsed peak current, powder } \\
\text { concentration, powder size }\end{array}$ & $\begin{array}{l}\text { Dimensional accuracy } \\
\text { and precision }\end{array}$ & {$[33]$} \\
\hline DP & AISI-1018 steel & V and FR & $\begin{array}{l}\text { Process zone } \\
\text { temperature, burr } \\
\text { formation, and chip } \\
\text { morphology }\end{array}$ & {$[34]$} \\
\hline LAJEM & WC-Co composite & $\begin{array}{l}\text { Inter-electrode gap, supply voltage, } \\
\text { electrolyte concentration, and duty cycle }\end{array}$ & $\begin{array}{l}\text { Hole taper angle, MRR, } \\
\text { and SQ }\end{array}$ & [35] \\
\hline $\mathrm{TP}$ & AISI-1015 steel & V, DoC, FR, coolant flow rate & TW and SQ & [36] \\
\hline $\mathrm{TP}$ & $\begin{array}{l}\mathrm{ZrSiO} 4 \text {-grade-LM25 } \\
\text { matrix composites }\end{array}$ & Coolant, V, FR, DoC & $\begin{array}{c}\text { Cutting forces, SQ, and } \\
\text { TW }\end{array}$ & [37] \\
\hline
\end{tabular}

Drilling process (DP), abrasive waterjet machining (AWJM), turning process (TP), electrical discharge machining (EDM), milling process (MP), laser machining (LM), speed (V), feed rate (FR), depth-of-cut (DoC), material removal rate (MRR), electrode wear rate (EWR), laser-assisted jet electro-chemical machining (LAJEM), surface quality (SQ), tool wear (TW). 
Ultrasonic vibration-assisted turning (UVAT) is a machining technique in which vibrations are imposed on the cutting insert, resulting in the intermittent cutting of materials [38]. UVAT has shown significant improvements in surface quality and a decline in the cutting forces in hard-to-cut materials $[19,39,40]$. In the current work, a fuzzy logic technique is adopted for the simulation of UVAT and the conventional turning (CT) processes of Ti-6Al7Zr3Nb4Mo0.9Nd to predict the cutting forces, maximum process zone temperature, chip compression ratio (CCR), surface roughness, and shear angle (SA). The fuzzy model was validated with experimental results and was used for the comparative analysis of UVAT and CT.

\section{Proposed Fuzzy Logic Algorithm}

\subsection{Fuzzy-Based Algorithms for UVAT and CT}

Figure 1 presents the conceptual illustration of the developed fuzzy inference systems for the calculation of the maximum process zone temperature, cutting forces, surface roughness, CCR, and SA in CT and UVAT at the selected range of input parameters.

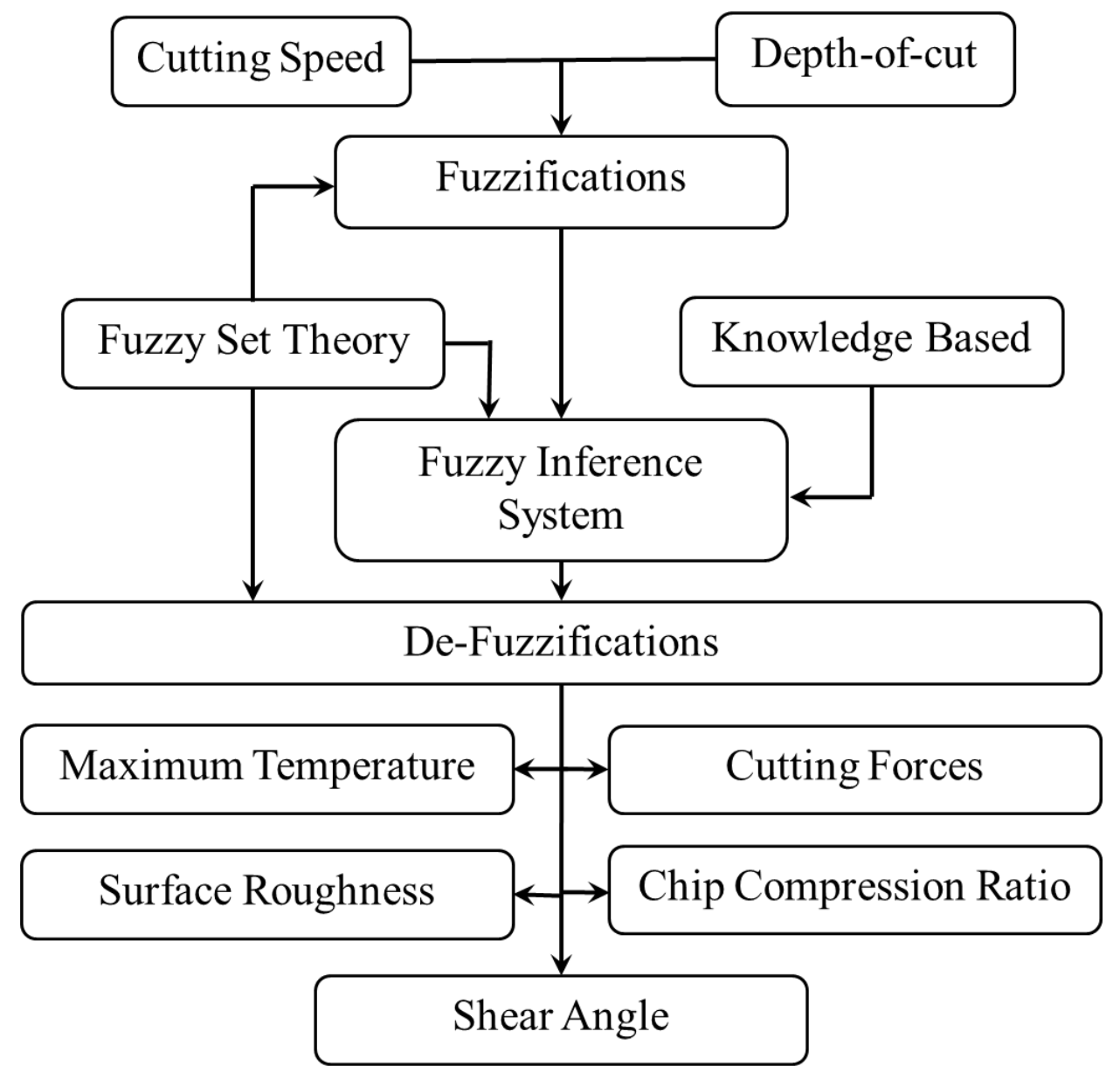

Figure 1. Schematic illustration of developed fuzzy inference systems for CT and UVAT.

The developed fuzzy inference systems are designed for two input variables, speed (V) and depth-of-cut (DoC), for the prediction of output variables such as maximum temperature, cutting forces, surface roughness, CCR, and SA during the CT and UVAT processes. The input variables are fed to the fuzzy plane, and the selected output variables are determined based on the defined fuzzy rules-based system. Moreover, in the developed algorithms, the output calculation of the scheme is established on the centroid-method, and de-fuzzification is accomplished using the Mamdani implication. The feed rate used in the current simulations were assumed to be constant at $0.1 \mathrm{~mm} / \mathrm{rev}$, whereas the axial 
force $(F a)$ component was also ignored due to its low level when compared to the radial force $(F r)$ and tangential force components $(F t)$. An amplitude of $10.0 \mu \mathrm{m}$ and a frequency of $20.0 \mathrm{kHz}$ were assumed in the developed UVAT process.

\subsubsection{Fuzzy Membership Functions}

A triangular membership function was used for the input (V and DoC) and output (tangential/radial forces, maximum process zone temperature, surface roughness, CCR, and SA) variables in the developed models based on the recommendation of Pedrycz [41]. The triangular function is mathematically represented in Equation (1):

$$
\operatorname{Variables}(u, m, v)=\left\{\begin{array}{cc}
0 & s \leq u \\
\frac{s-u}{m-s} & u<s \leq m \\
\frac{v-s}{v-m} & m<s \leq v \\
0 & s \geq v
\end{array}\right.
$$

where $u, m$, and $v$ represent triangular membership function. Details can be found elsewhere [21].

\subsubsection{Fuzzy Sets Used in Simulation}

The two input variables $\mathrm{V}$ and DoC were divided into 9 fuzzy sets, which were represented as very-very-low (VVL), very-low (VL), low (L), medium-low (ML), medium (M), Medium-high $(\mathrm{MH})$, high $(\mathrm{H})$, very-high $(\mathrm{VH})$, and very-very-high $(\mathrm{VVH})$ in both the CT and UVAT in the developed fuzzy inference systems. The feed rate in the developed models was assumed to be constant at $0.1 \mathrm{~mm} / \mathrm{rev}$. Additionally, the output variables were distributed into 16 fuzzy-sets, i.e., extremely-low (EL), very-very-very-low (VVVL), very-very-low (VVL), very-low (VL), low (L), medium-low-low (MLL), medium-low (ML), medium (M), medium-high $(\mathrm{MH})$, medium-high-high $(\mathrm{MHH})$, high $(\mathrm{H})$, very-high $(\mathrm{VH})$, very-very-high (VVH), very-very-very-high (VVVH), and extremely-high (EH). The defined input variables with their related membership functions are given in Figure 2 . The output variables for the UVAT and CT systems with their associated membership functions are presented in Figures 3 and 4, respectively. Additionally, additional information and terminology considered for the defined input and output fuzzy sets used in the simulations of the CT and UVAT processes are listed in Tables 2 and 3, respectively.
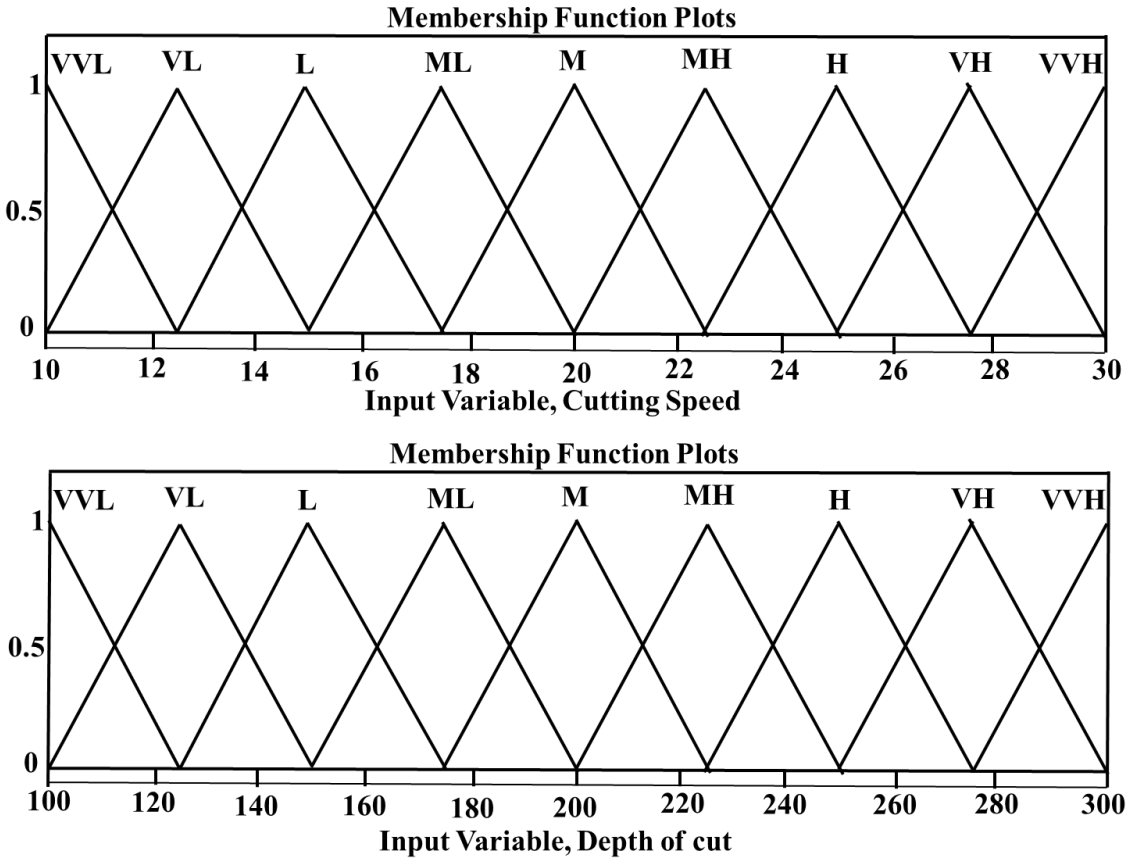

Figure 2. Input variables with associated membership functions used in the simulation of UVAT and CT. 

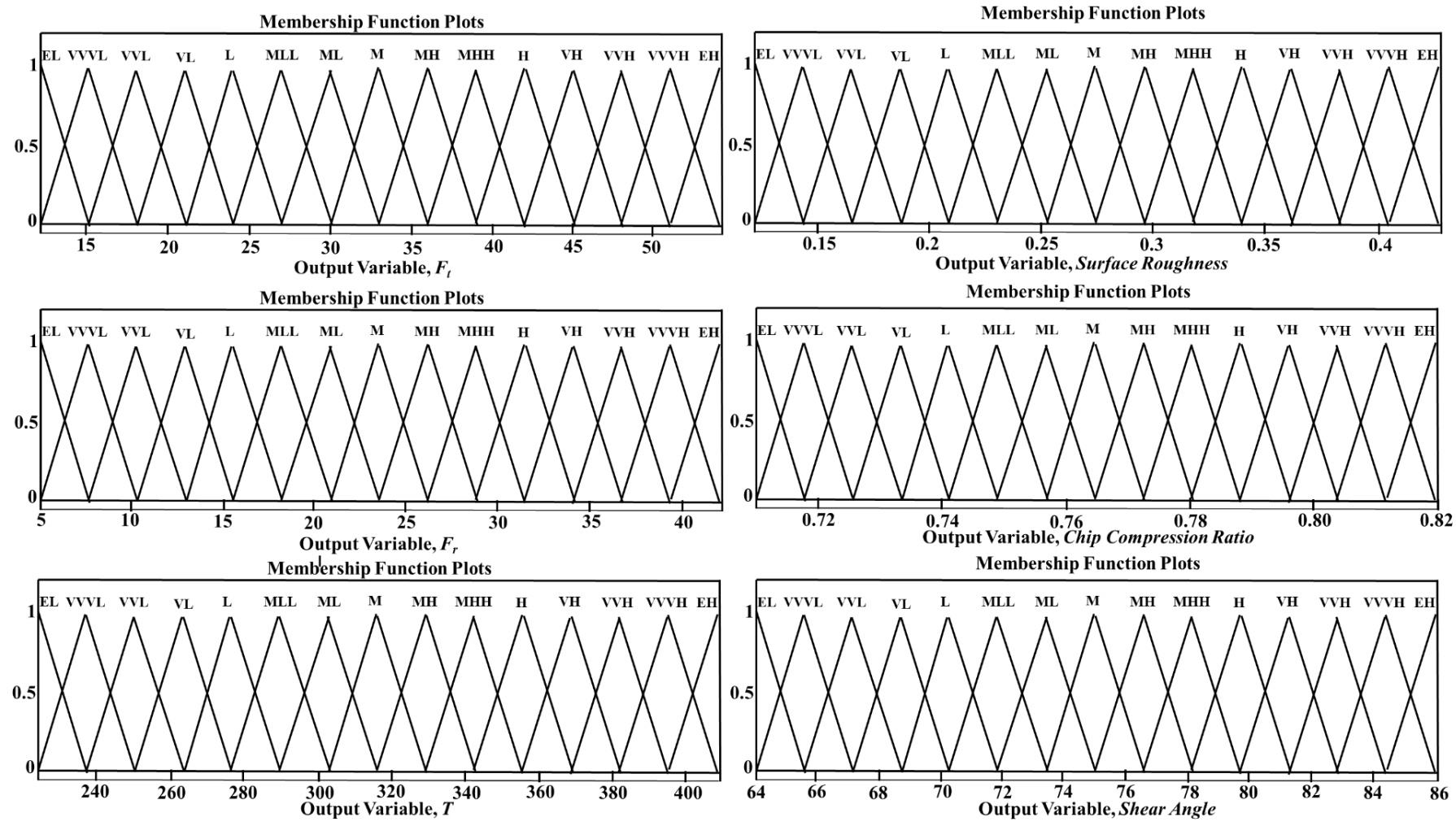

Figure 3. Output variables with associated membership functions used in the simulation of UVAT.
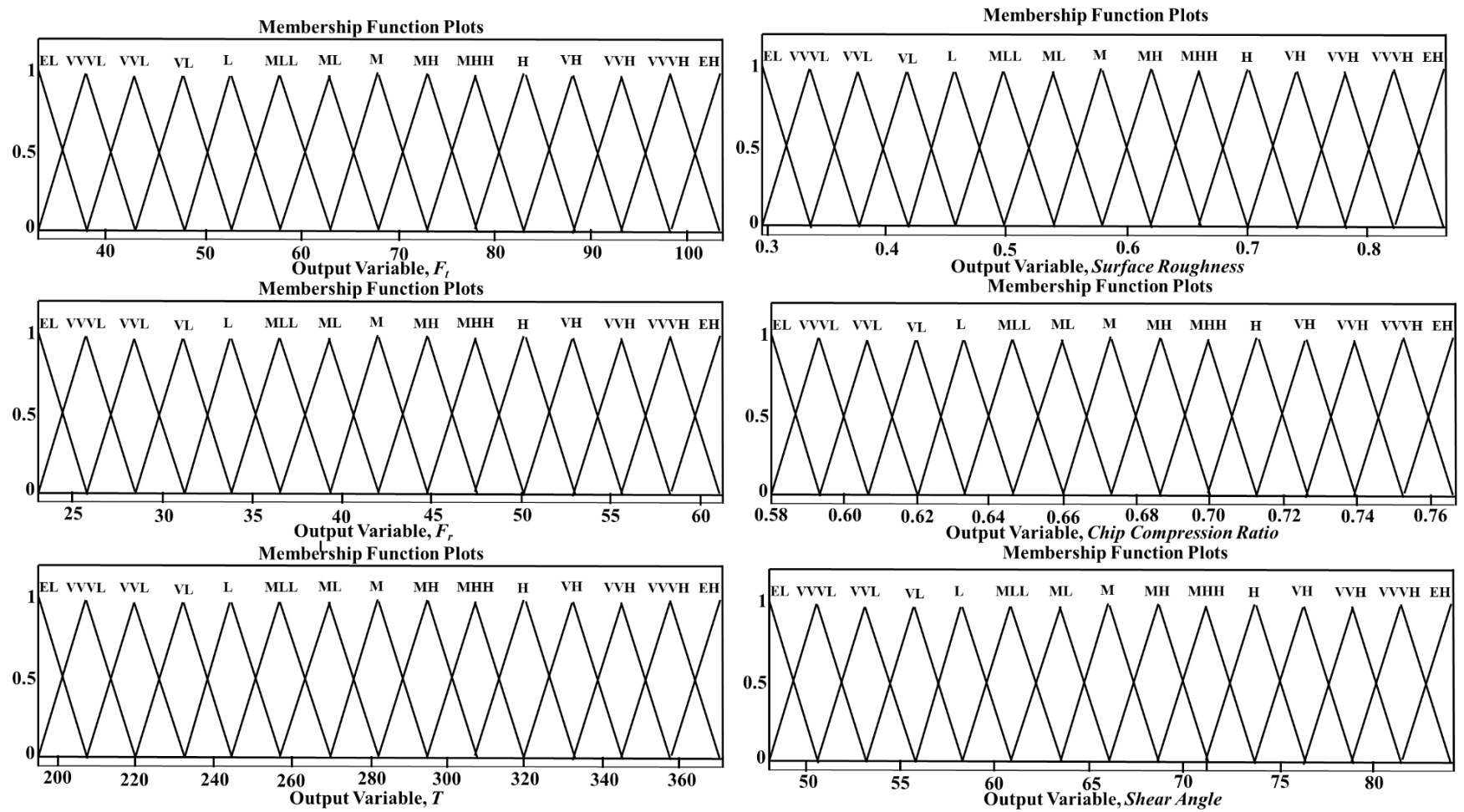

Figure 4. Output variables with associated membership functions used in the simulation of CT.

\subsubsection{Proposed Rules}

The set of rules were defined for both the developed fuzzy inference systems to calculate the output variables against the defined combinations of the input variables within the defined range, as represented in Tables 4 and 5 for UVAT and CT, respectively. 
Table 2. Input and output parameters used in the CT fuzzy inference system.

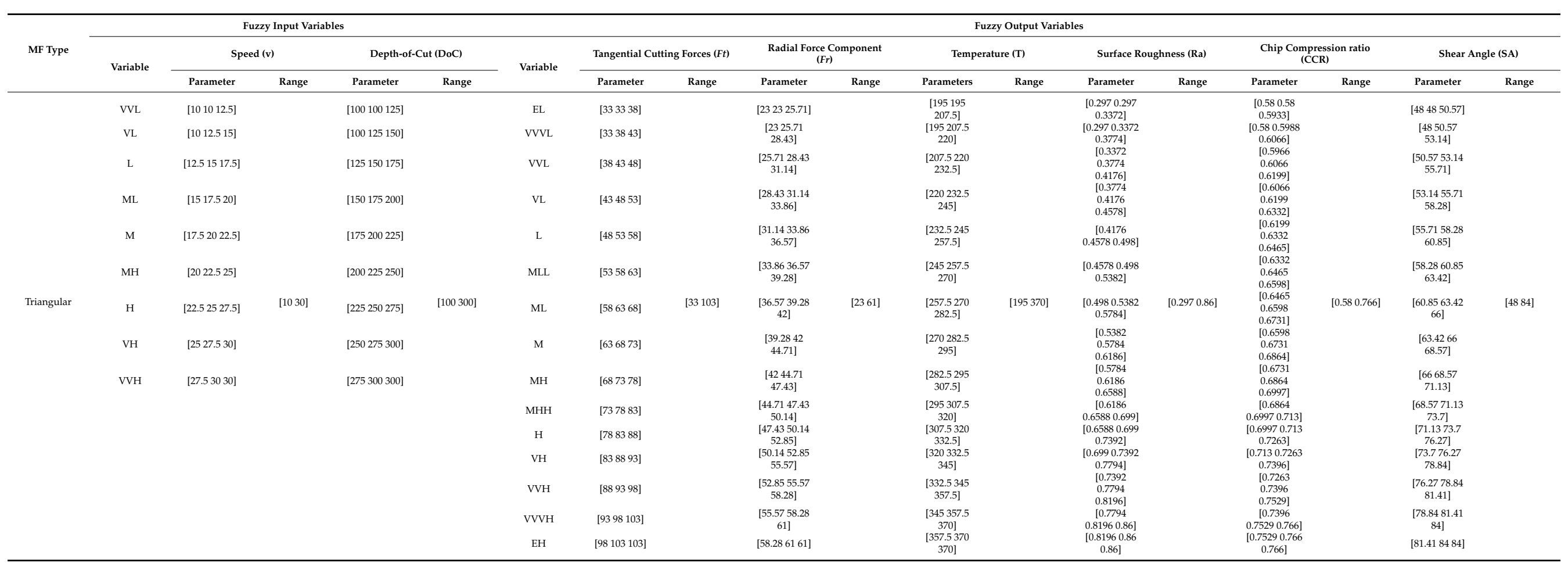


Table 3. Input and output parameters used in the UVAT fuzzy inference system.

\begin{tabular}{|c|c|c|c|c|c|c|c|c|c|c|c|c|c|c|c|c|c|c|}
\hline \multirow{3}{*}{ MF Type } & \multicolumn{5}{|c|}{ Fuzzy Input Variables } & \multicolumn{13}{|c|}{ Fuzzy Output Variables } \\
\hline & \multirow{2}{*}{ Variable } & \multicolumn{2}{|c|}{ Speed $(v)$} & \multicolumn{2}{|c|}{ Depth-of-Cut (DoC) } & \multirow{2}{*}{ Variable } & \multicolumn{2}{|c|}{ Tangential Cutting Forces $(F t)$} & \multicolumn{2}{|c|}{$\begin{array}{l}\text { Radial Force Component } \\
(F r)\end{array}$} & \multicolumn{2}{|c|}{ Temperature (T) } & \multicolumn{2}{|c|}{ Surface Roughness (Ra) } & \multicolumn{2}{|c|}{$\begin{array}{l}\text { Chip Compression ratio } \\
\text { (CCR) }\end{array}$} & \multicolumn{2}{|c|}{ Shear Angle (SA) } \\
\hline & & Parameter & Range & Parameter & Range & & Parameter & Range & Parameter & Range & Parameters & Range & Parameter & Range & Parameter & Range & Parameter & Range \\
\hline \multirow{13}{*}{ Triangular } & vVL & {$\left[\begin{array}{llll}10 & 10 & 12.5\end{array}\right]$} & \multirow{13}{*}[1030]{} & [100 100 125] & \multirow{13}{*}[100300]{} & $\mathrm{EL}$ & [12 12 15] & \multirow{13}{*}{ [12 54] } & {$[557.65]$} & \multirow{13}{*}{ [5 42] } & {$[224224$} & \multirow{13}{*}{ [224 408] } & $\begin{array}{l}{[0.1210 .121} \\
0.143]\end{array}$ & \multirow{13}{*}{ [0.121 0.429] } & {$[0.710 .71$} & \multirow{13}{*}[0.710.8122]{} & [64 64 65.57] & \multirow{13}{*}{ [64 86] } \\
\hline & $\mathrm{L}$ & [12.5 15 17.5] & & [125 150 175] & & VVL & $151821]$ & & $\begin{array}{l}{[7.6510 .3} \\
12.95]\end{array}$ & & $\begin{array}{c}{[237.2250 .4} \\
263.6]\end{array}$ & & $\begin{array}{c}{[0.1430 .165} \\
0.187]\end{array}$ & & $\begin{array}{l}{[0.7173} \\
0.7746 \\
0.7319]\end{array}$ & & $\begin{array}{c}{[65.5767 .14} \\
68.71]\end{array}$ & \\
\hline & $\mathrm{ML}$ & {$[1517.520]$} & & [150 175 200] & & $\mathrm{VL}$ & [182124] & & $\begin{array}{c}{[10.312 .95} \\
15.6]\end{array}$ & & $\begin{array}{l}{[250.4263 .6} \\
276.8]\end{array}$ & & $\begin{array}{c}{[0.1650 .187} \\
0.209]\end{array}$ & & $\begin{array}{c}{[0.7246} \\
0.7319 \\
0.7392]\end{array}$ & & $\begin{array}{c}{[67.1468 .71} \\
70.28]\end{array}$ & \\
\hline & M & [17.5 2022.5] & & [175 200 225] & & $\mathrm{L}$ & [21 24 27] & & $\begin{array}{c}{[12.9515 .6} \\
18.25]\end{array}$ & & $\begin{array}{l}{[263.6276 .8} \\
290]\end{array}$ & & $\begin{array}{c}{\left[\begin{array}{c}0.1870 .209 \\
0.231]\end{array}\right.} \\
\end{array}$ & & $\begin{array}{c}{[0.7319} \\
0.7392 \\
0.7465]\end{array}$ & & $\begin{array}{c}{[68.7170 .28} \\
77.85]\end{array}$ & \\
\hline & мH & [20 22.525] & & [200 225 250] & & MLL & [24 27 30] & & $\begin{array}{l}{[15.618 .25} \\
20.9]\end{array}$ & & $\begin{array}{c}{[276.8290} \\
303.2]\end{array}$ & & {$\left[\begin{array}{c}{[0.20990 .231} \\
0.253]\end{array}\right.$} & & $\begin{array}{c}{[0.7392} \\
0.7655 \\
0.7538]\end{array}$ & & $\begin{array}{c}{[70.2871 .85} \\
73.42]\end{array}$ & \\
\hline & $\mathrm{H}$ & [22.5 25 27.5] & & [225 250 275] & & $\mathrm{ML}$ & [27 30 33] & & $\begin{array}{c}{[18.2520 .9} \\
23.55]\end{array}$ & & $\begin{array}{l}{[2900303.2} \\
316.4]\end{array}$ & & {$\left[\begin{array}{l}{[0.2310 .253} \\
0.275]\end{array}\right.$} & & $\begin{array}{l}{[0.76165} \\
0.7538 \\
0.7611]\end{array}$ & & $\begin{array}{c}{[71.8573 .42} \\
75]\end{array}$ & \\
\hline & vH & [25 27.530] & & [250 275 300] & & M & [30 33 36] & & $\begin{array}{c}{[20.923 .55} \\
26.2]\end{array}$ & & $\begin{array}{c}{[303.2316 .4} \\
329.6]\end{array}$ & & $\begin{array}{c}{[0.2530 .275} \\
0.297]\end{array}$ & & $\begin{array}{l}{[0.7538} \\
0.7611 \\
0.7684]\end{array}$ & & $\begin{array}{l}{[77.42 .7275} \\
76.57]\end{array}$ & \\
\hline & \multirow[t]{6}{*}{ VVH } & \multirow[t]{6}{*}{ [27.5 3030] } & & \multirow[t]{6}{*}{ [275 300 300] } & & MH & [33 36 39] & & $\begin{array}{l}{[23.5526 .2} \\
28.85]\end{array}$ & & $\underset{342.81}{[316.4329 .6}$ & & $\begin{array}{c}{[0.2750 .297} \\
0.319]\end{array}$ & & $\begin{array}{l}{[0.7611} \\
0.7684 \\
0.7757]\end{array}$ & & $\begin{array}{c}{[7576.57} \\
78.13]\end{array}$ & \\
\hline & & & & & & MHH & [36 39 42] & & $\begin{array}{l}{[26.228 .85} \\
31.5]\end{array}$ & & $\begin{array}{l}{[329.6342 .8} \\
356]\end{array}$ & & {$\left[\begin{array}{l}{[0.2970 .319} \\
0.341]\end{array}\right.$} & & $\begin{array}{c}0.7684 \\
0.77570 .783]\end{array}$ & & $\begin{array}{l}{[76.5778 .13} \\
79.7]\end{array}$ & \\
\hline & & & & & & $\mathrm{H}$ & [39 42 45] & & {$[28.8531 .5$} & & $\begin{array}{l}{[342.2356} \\
3692.2]\end{array}$ & & {$\left[\begin{array}{l}{[0.3190 .341} \\
0.363]\end{array}\right.$} & & {$[0.77507 .783$} & & $\begin{array}{l}{[78.1379 .7} \\
81.27]\end{array}$ & \\
\hline & & & & & & VVH & [45 48 51] & & $\begin{array}{c}{[34.1536 .8} \\
39.45]\end{array}$ & & $\begin{array}{c}{[369.2382 .4} \\
395.6]\end{array}$ & & $\begin{array}{c}{[0.3630 .385} \\
0.407]\end{array}$ & & $\begin{array}{l}{[0.7903} \\
0.7976 \\
0.8049]\end{array}$ & & $\begin{array}{c}{[81.2782 .84} \\
84.41]\end{array}$ & \\
\hline & & & & & & VVVH & [48 51 54] & & $\underset{42]}{[36.839 .45}$ & & $\begin{array}{l}{[382.4395 .6} \\
408]\end{array}$ & & $\begin{array}{l}{[0.3850 .407} \\
0.429]\end{array}$ & & $\begin{array}{c}{[0.7976} \\
0.8049 \\
0.8122]\end{array}$ & & $\begin{array}{l}{[82.8484 .41} \\
86]\end{array}$ & \\
\hline & & & & & & $\mathrm{EH}$ & [51 54 54] & & [39.45 42 42] & & $\begin{array}{l}{[395.6408 .8} \\
408]\end{array}$ & & $\begin{array}{l}{[0.4070 .429} \\
0.429]\end{array}$ & & $\begin{array}{l}{[0.8049} \\
0.8122 \\
0.8122]\end{array}$ & & [84.41 86 86] & \\
\hline
\end{tabular}


Table 4. The rules used in the UVAT simulation.

\begin{tabular}{|c|c|c|c|c|c|c|c|c|c|c|c|c|c|c|c|c|c|}
\hline & & & & & & & & & & & & & & & & & \\
\hline Rules No & Speed & Depth-of-Cut & $\begin{array}{c}\text { Tangential } \\
\text { Cutting Force }\end{array}$ & $\begin{array}{c}\text { Radial } \\
\text { Cutting Force } \\
\end{array}$ & Temperature & $\begin{array}{c}\text { Surface } \\
\text { Roughness }\end{array}$ & $\begin{array}{c}\text { Chip } \\
\text { Compression } \\
\text { Ratio (CCR) } \\
\end{array}$ & $\begin{array}{c}\text { Shear Angle } \\
\text { (SA) }\end{array}$ & Rules No & Speed & Depth-of-Cut & $\begin{array}{c}\text { Tangential } \\
\text { Cutting Force }\end{array}$ & $\begin{array}{c}\text { Radial } \\
\text { Cutting Force }\end{array}$ & Temperature & $\begin{array}{c}\begin{array}{c}\text { Surface } \\
\text { Roughness }\end{array} \\
\end{array}$ & $\begin{array}{c}\text { Chip } \\
\text { Compression } \\
\text { Ratio (CCR) }\end{array}$ & $\begin{array}{c}\text { Shear Angle } \\
\text { (SA) }\end{array}$ \\
\hline R1 & VVL & VVL & $\begin{array}{c}\mathrm{EL} \\
\mathrm{EW}\end{array}$ & EL & EL & ML & $\begin{array}{c}\mathrm{EL} \\
\mathrm{J}\end{array}$ & $\mathrm{MH}$ & R2 & VVL & $\mathrm{VL}$ & VVVL & VVVL & VVVL & M & $\mathrm{EL}$ & MHH \\
\hline R3 & $\mathrm{VVL}$ & $\mathrm{L}$ & VVL & VVL & VVVL & MHH & VVVL & $\mathrm{H}$ & $\mathrm{R} 4$ & VVL & ML & & & VVL & $\mathrm{VH}$ & VVVL & VVH \\
\hline $\begin{array}{l}\text { R5 } \\
\text { R7 }\end{array}$ & $\begin{array}{l}\text { VVL } \\
\text { VVL }\end{array}$ & $\underset{\mathrm{H}}{\mathrm{M}}$ & $\begin{array}{l}\mathrm{L} \\
\mathrm{ML}\end{array}$ & $\begin{array}{l}\mathrm{L} \\
\mathrm{ML}\end{array}$ & $\begin{array}{l}\text { VL } \\
\text { MLL }\end{array}$ & $\begin{array}{l}\text { VVH } \\
\text { VVVH }\end{array}$ & VVL & $\begin{array}{l}\text { VVVH } \\
\text { VVVH }\end{array}$ & $\begin{array}{l}\text { R6 } \\
\text { R8 }\end{array}$ & vVL & $\mathrm{MH}$ & MLL & MLL & L & $\mathrm{VVVH}$ & VVL & $\mathrm{VVVH}$ \\
\hline $\begin{array}{l}\text { R7 } \\
\text { R9 }\end{array}$ & $\begin{array}{l}\text { VVL } \\
\text { VVL }\end{array}$ & $\begin{array}{c}\mathrm{H} \\
\mathrm{VVH}\end{array}$ & $\begin{array}{l}\mathrm{ML} \\
\mathrm{MH}\end{array}$ & $\begin{array}{l}\mathrm{ML} \\
\mathrm{MH}\end{array}$ & $\begin{array}{l}\text { MLL } \\
\text { ML }\end{array}$ & $\mathrm{EH}$ & $\begin{array}{l}\text { MLL } \\
\text { MLL }\end{array}$ & $\mathrm{EH}$ & $\begin{array}{l}\text { R8 } \\
\text { R10 }\end{array}$ & $\begin{array}{ll}\mathrm{VL} \\
\mathrm{VL}\end{array}$ & $\begin{array}{l}\mathrm{VH} \\
\mathrm{VVL}\end{array}$ & $\begin{array}{c}\mathrm{M} \\
\mathrm{VVVL}\end{array}$ & $\begin{array}{c}\mathrm{M} \\
\mathrm{VVVL}\end{array}$ & $\begin{array}{l}\text { MLL } \\
\text { VVVL }\end{array}$ & $\begin{array}{l}\mathrm{EH} \\
\text { MLL }\end{array}$ & $\begin{array}{l}\text { MLL } \\
\text { VVVL }\end{array}$ & $\begin{array}{l}\text { EH } \\
\text { MHH }\end{array}$ \\
\hline R11 & $\mathrm{VL}$ & $\mathrm{VL}$ & $\begin{array}{l}\mathrm{Mn} \\
\mathrm{VVL}\end{array}$ & 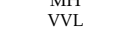 & $\begin{array}{l}\text { NVVL } \\
\text { VVVL }\end{array}$ & $\mathrm{M}$ & VVL & ${ }_{\mathrm{H}}^{\mathrm{Ln}}$ & R12 & $\mathrm{VL}$ & L & $\mathrm{VL}$ & $\mathrm{VL}$ & VVL & MHH & VVL & $\mathrm{VH}$ \\
\hline R13 & $\mathrm{VL}$ & ML & $\mathrm{L}$ & $\mathrm{L}$ & $\mathrm{VL}$ & $\mathrm{VH}$ & $\mathrm{VL}$ & $\mathrm{VVH}$ & R14 & $\mathrm{VL}$ & $\mathrm{M}$ & MLL & MLL & $\mathrm{L}$ & $\mathrm{VH}$ & $\mathrm{VL}$ & $\mathrm{VVVH}$ \\
\hline R15 & $\mathrm{VL}$ & MH & ML & $\mathrm{ML}$ & MLL & $\mathrm{VVH}$ & $\mathrm{VL}$ & VVVH & R16 & ML & $\mathrm{H}$ & $\mathrm{M}$ & $\mathrm{M}$ & MLL & $\mathrm{VVH}$ & $\mathrm{L}$ & EH \\
\hline $\begin{array}{l}\text { R17 } \\
\text { R19 }\end{array}$ & $\begin{array}{l}\mathrm{VL} \\
\mathrm{L}\end{array}$ & $\begin{array}{l}\mathrm{VH} \\
\mathrm{VVL}\end{array}$ & $\begin{array}{l}\mathrm{MH} \\
\mathrm{VVL}\end{array}$ & $\begin{array}{l}\mathrm{MH} \\
\mathrm{VVL}\end{array}$ & $\begin{array}{l}\mathrm{ML} \\
\mathrm{VVL}\end{array}$ & $\begin{array}{l}\text { VVVH } \\
\text { MLL }\end{array}$ & $\begin{array}{l}\text { MLL } \\
\text { VVL }\end{array}$ & $\underset{\mathrm{H}}{\mathrm{EH}}$ & R18 & VL & $\mathrm{VVH}$ & MHH & MHH & $\mathrm{M}$ & EH & MLL & EH \\
\hline $\begin{array}{l}\text { R219 } \\
\text { R21 }\end{array}$ & ${ }_{\mathrm{L}}^{\mathrm{L}}$ & $\mathrm{W}$ & $\begin{array}{ll}\mathrm{VWL} \\
\mathrm{L}\end{array}$ & $\mathrm{WL}$ & $\mathrm{VL}$ & ${ }_{M}^{\mathrm{MLL}}$ & $\mathrm{VL}_{\mathrm{VL}}$ & $\begin{array}{c}\mathrm{H} \\
\mathrm{VH}\end{array}$ & $\begin{array}{l}\begin{array}{l}200 \\
\text { R22 }\end{array} \\
\text {. }\end{array}$ & $\begin{array}{l}\mathrm{L} \\
\mathrm{L}\end{array}$ & $\begin{array}{l}\mathrm{VL} \\
\mathrm{ML}\end{array}$ & $\begin{array}{l}\mathrm{VL} \\
\text { MLL }\end{array}$ & $\begin{array}{l}\mathrm{VL} \\
\mathrm{MLL}\end{array}$ & $\mathrm{WL}$ & $\begin{array}{c}\mathrm{ML} \\
\mathrm{H}\end{array}$ & $\begin{array}{c}\mathrm{VL} \\
\mathrm{L}\end{array}$ & $\begin{array}{c}\mathrm{H} \\
\mathrm{VVVH}\end{array}$ \\
\hline R23 & $\begin{array}{l}\mathrm{L} \\
\mathrm{L}\end{array}$ & M & $\begin{array}{l}\mathrm{L} \\
\mathrm{ML}\end{array}$ & $\begin{array}{l}\mathrm{L} \\
\mathrm{ML}\end{array}$ & MLL & $\begin{array}{l}\mathrm{M} \\
\mathrm{H}\end{array}$ & $\mathrm{V}_{\mathrm{L}}$ & VVVH & R24 & ${ }_{\mathrm{L}}^{\mathrm{L}}$ & MH & $\mathrm{M}$ & $\mathrm{M}$ & $\begin{array}{l}\mathrm{L} \\
\mathrm{ML}\end{array}$ & $\mathrm{rH}$ & MLL & VVVH \\
\hline $\mathrm{R} 25$ & L & $\mathrm{H}$ & $\mathrm{MH}$ & $\mathrm{M}$ & M & $\mathrm{vH}$ & MLL & $\mathrm{EH}$ & R26 & $\mathrm{L}$ & $\mathrm{VH}$ & MHH & $\mathrm{M}$ & $\mathrm{MH}$ & VVVH & $\mathrm{ML}$ & $\mathrm{EH}$ \\
\hline R27 & $\mathrm{L}$ & VVH & $\mathrm{H}$ & MHH & MH & EH & $\mathrm{ML}$ & EH & R28 & ML & VVL & VVL & VVL & VVL & L & EL & $\mathrm{L}$ \\
\hline R29 & $\begin{array}{l}\text { ML } \\
\text { ML }\end{array}$ & $\begin{array}{l}\mathrm{VL} \\
\mathrm{ML}\end{array}$ & $\mathrm{VL}_{\mathrm{L}}$ & $\underset{\mathrm{L}}{\mathrm{VL}}$ & $\begin{array}{l}\mathrm{VL} \\
\mathrm{MLL}\end{array}$ & $\begin{array}{c}\text { M } \\
\text { MHH }\end{array}$ & $\mathrm{VVVL}$ & MLL & R30 & ML & $\mathrm{L}$ & VL & VL & $\mathrm{L}$ & $\mathrm{MH}$ & VVL & ML \\
\hline $\begin{array}{l}\text { R31 } \\
\text { R33 }\end{array}$ & $\begin{array}{l}\mathrm{ML} \\
\mathrm{ML}\end{array}$ & $\begin{array}{l}\mathrm{ML} \\
\mathrm{MH}\end{array}$ & $\begin{array}{c}\mathrm{L} \\
\mathrm{ML}\end{array}$ & $\begin{array}{l}\mathrm{L} \\
\mathrm{ML}\end{array}$ & $\begin{array}{l}\text { MLL } \\
\text { ML }\end{array}$ & $\mathrm{MHH}_{\mathrm{H}}$ & $\begin{array}{l}\mathrm{VL} \\
\mathrm{ML}\end{array}$ & $\begin{array}{l}\mathrm{M} \\
\mathrm{MH}\end{array}$ & $\begin{array}{l}\text { R332 } \\
\text { 334 }\end{array}$ & $\begin{array}{l}\mathrm{ML} \\
\mathrm{ML}\end{array}$ & $\begin{array}{c}\mathrm{M} \\
\mathrm{H}\end{array}$ & $\begin{array}{l}\text { MLL } \\
\text { M }\end{array}$ & ${ }_{\mathrm{M}}^{\mathrm{MLL}}$ & ${ }_{M}^{M L L}$ & $\begin{array}{l}\mathrm{H} \\
\mathrm{H}\end{array}$ & $\begin{array}{c}\mathrm{L} \\
\mathrm{MH}\end{array}$ & $\begin{array}{l}\mathrm{MH} \\
\mathrm{MHH}\end{array}$ \\
\hline $\begin{array}{l}\text { R35 } \\
\text { R }\end{array}$ & ML & VH & M & $\begin{array}{l}\mathrm{ML} \\
\mathrm{MH}\end{array}$ & $\begin{array}{l}\mathrm{ML} \\
\mathrm{MH}\end{array}$ & $\begin{array}{c}\mathrm{H} \\
\text { VVH }\end{array}$ & $\begin{array}{l}\mathrm{ML} \\
\mathrm{MHH}\end{array}$ & H & $\begin{array}{l}\text { R34 } \\
\text { R36 }\end{array}$ & $\begin{array}{l}\text { ML } \\
\text { ML }\end{array}$ & VVH & MHH & MHH & MHH & $\begin{array}{r}\mathrm{H} \\
\mathrm{VVVH}\end{array}$ & H & $\mathrm{H}$ \\
\hline R37 & M & VVL & $\mathrm{VL}$ & VVL & $\mathrm{L}$ & $\mathrm{VL}$ & $\mathrm{EL}$ & MLL & R38 & M & $\mathrm{VL}$ & $\mathrm{L}$ & $\mathrm{VL}$ & L & L & VVL & $\mathrm{ML}$ \\
\hline R39 & $\mathrm{M}$ & $\mathrm{L}$ & MLL & $\mathrm{L}$ & MLL & ML & $\begin{array}{l}\mathrm{VL} \\
\mathrm{NI}\end{array}$ & M & R40 & M & ML & ML & MLL & MLL & $\mathrm{M}$ & $\mathrm{L}$ & $\mathrm{MH}$ \\
\hline $\begin{array}{l}\text { R41 } \\
\text { R }\end{array}$ & $\mathrm{M}$ & M & M & ML & ML & MHH & ML & MHH & $\begin{array}{r}\text { R42 } \\
\text { R44 }\end{array}$ & $\mathrm{M}$ & $\mathrm{MH}$ & $\mathrm{MH}$ & M & $\begin{array}{c}\mathrm{M} \\
\mathrm{MHH}\end{array}$ & $\mathrm{H}$ & $\mathrm{M}$ & MHH \\
\hline $\begin{array}{l}\text { R43 } \\
\text { R45 }\end{array}$ & $\begin{array}{l}\mathrm{M} \\
\mathrm{M}\end{array}$ & $\begin{array}{l}\mathrm{H} \\
\mathrm{VVH}\end{array}$ & $\begin{array}{l}\mathrm{MHH} \\
\mathrm{VH}\end{array}$ & $\begin{array}{c}\mathrm{MH} \\
\mathrm{H}\end{array}$ & $\begin{array}{c}\mathrm{MH} \\
\mathrm{H}\end{array}$ & $\begin{array}{c}\mathrm{H} \\
\mathrm{VVH}\end{array}$ & $\begin{array}{l}\mathrm{MHH} \\
\mathrm{vH}\end{array}$ & $\begin{array}{l}\mathrm{H} \\
\mathrm{VH}\end{array}$ & $\begin{array}{l}\text { R44 } \\
\text { R46 }\end{array}$ & $\begin{array}{l}\mathrm{M} \\
\mathrm{MH}\end{array}$ & $\begin{array}{l}\mathrm{VH} \\
\mathrm{VVL}\end{array}$ & $\begin{array}{l}\mathrm{H} \\
\mathrm{L}\end{array}$ & $\begin{array}{c}\mathrm{MHH} \\
\mathrm{VL}\end{array}$ & $\begin{array}{c}\mathrm{MHH} \\
\mathrm{L}\end{array}$ & $\begin{array}{l}\mathrm{VH} \\
\mathrm{VL}\end{array}$ & $\begin{array}{c}\mathrm{H} \\
\mathrm{VVVL}\end{array}$ & $\begin{array}{l}\mathrm{VH} \\
\mathrm{ML}\end{array}$ \\
\hline $\begin{array}{l}\text { R45 } \\
\text { R47 }\end{array}$ & $\begin{array}{l}\mathrm{M} \\
\mathrm{MH}\end{array}$ & $\mathrm{VL}$ & $\underset{\mathrm{L}}{\mathrm{VH}}$ & $\begin{array}{l}\mathrm{H} \\
\mathrm{VL}\end{array}$ & MLL & $\mathrm{L}$ & $\begin{array}{l}\text { VH } \\
\text { VVL }\end{array}$ & $\mathrm{M}$ & 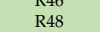 & $\mathrm{MH}$ & ${ }_{\mathrm{L}}$ & MLL & 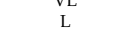 & $\begin{array}{l}\mathrm{L} \\
\mathrm{ML}\end{array}$ & $\begin{array}{l}\mathrm{V} \\
\mathrm{L}\end{array}$ & $\mathrm{VL}$ & MH \\
\hline R49 & $\mathrm{MH}$ & ML & ML & MLL & ML & ML & MLL & MHH & $\begin{array}{l}\text { R50 } \\
\text { R }\end{array}$ & $\mathrm{MH}$ & $\mathrm{M}$ & $\mathrm{M}$ & $\mathrm{ML}$ & $\mathrm{M}$ & $\mathrm{M}$ & ML & $\mathrm{H}$ \\
\hline R51 & MH & MH & MH & MH & MH & MH & MH & H & R52 & MH & $\mathrm{H}$ & MHH & MHH & MHH & MHH & $\mathrm{H}$ & $\mathrm{VH}$ \\
\hline $\begin{array}{l}\text { R53 } \\
\text { R55 }\end{array}$ & $\mathrm{MH}$ & VH & $\mathrm{H}$ & MHH & $\mathrm{H}$ & H & VH & VVH & $\begin{array}{l}\text { R54 } \\
\text { P55 }\end{array}$ & $\mathrm{MH}$ & $\mathrm{VVH}$ & $\mathrm{VH}$ & VH & VH & VVH & VH & VVH \\
\hline $\begin{array}{l}\text { R55 } \\
\text { R57 }\end{array}$ & $\begin{array}{l}\mathrm{H} \\
\mathrm{H}\end{array}$ & $\begin{array}{c}\mathrm{V} \\
\mathrm{V}\end{array}$ & $\begin{array}{l}\mathrm{L} \\
\mathrm{ML}\end{array}$ & $\begin{array}{l}\mathrm{VL} \\
\mathrm{L}\end{array}$ & $\begin{array}{l}\text { L } \\
\text { MLL }\end{array}$ & $\begin{array}{l}\text { VLL } \\
\text { VL }\end{array}$ & $\begin{array}{c}\text { VVVL } \\
\text { VL }\end{array}$ & $\begin{array}{l}\mathrm{EL} \\
\mathrm{VL}\end{array}$ & $\begin{array}{l}\text { R56 } \\
\text { R58 }\end{array}$ & $\begin{array}{l}\mathrm{H} \\
\mathrm{H}\end{array}$ & $\begin{array}{l}\mathrm{VL} \\
\mathrm{ML}\end{array}$ & $\begin{array}{l}\text { MLL } \\
M\end{array}$ & $\begin{array}{l}\text { VL } \\
\text { MLL }\end{array}$ & $\begin{array}{l}\mathrm{L} \\
\mathrm{ML}\end{array}$ & $\begin{array}{c}\text { VVL } \\
\text { L }\end{array}$ & $\begin{array}{c}\mathrm{VVL} \\
\mathrm{L}\end{array}$ & $\begin{array}{l}\text { VVVL } \\
\text { MLL }\end{array}$ \\
\hline R59 & $\begin{array}{l}\mathrm{H} \\
\mathrm{H}\end{array}$ & $\begin{array}{l}\mathrm{L} \\
\mathrm{M}\end{array}$ & $\mathrm{M}$ & $\begin{array}{l}\mathrm{L} \\
\mathrm{ML}\end{array}$ & $\mathrm{M}$ & $\begin{array}{l}\text { ML } \\
\text { MLL }\end{array}$ & $\begin{array}{c}\mathrm{V}_{\mathrm{L}} \\
\mathrm{c}\end{array}$ & $\begin{array}{l}\mathrm{VL} \\
\mathrm{ML}\end{array}$ & $\begin{array}{l}158 \\
\mathrm{R} 60\end{array}$ & $\begin{array}{l}\mathrm{H} \\
\mathrm{H}\end{array}$ & MH & $\begin{array}{l}\mathrm{M} \\
\mathrm{MH}\end{array}$ & M & $\begin{array}{l}\text { ML } \\
\text { MH }\end{array}$ & $\begin{array}{l}\mathrm{L} \\
\mathrm{M}\end{array}$ & $\begin{array}{l}\mathrm{L} \\
\mathrm{ML}\end{array}$ & ML \\
\hline R61 & $\mathrm{H}$ & $\mathrm{H}$ & MHH & MH & $\mathrm{H}$ & MH & $\mathrm{M}$ & M & $\begin{array}{l}\text { R662 } \\
\text { n }\end{array}$ & $\mathrm{H}$ & $\mathrm{VH}$ & $\mathrm{H}$ & MHH & $\mathrm{VH}$ & $\mathrm{MH}$ & $\mathrm{H}$ & MH \\
\hline $\begin{array}{l}\mathrm{R} 63 \\
\mathrm{R}\end{array}$ & $\mathrm{H}$ & VVH & $\mathrm{VH}$ & VH & VVH & VH & $\mathrm{VH}$ & MH & R64 & $\mathrm{VH}$ & VVL & $\mathrm{L}$ & L & ML & VVVL & VVL & VVVL \\
\hline $\begin{array}{l}\text { R65 } \\
\text { R67 }\end{array}$ & $\begin{array}{l}\mathrm{VH} \\
\mathrm{VH}\end{array}$ & $\begin{array}{l}\mathrm{VL} \\
\mathrm{ML}\end{array}$ & $\mathrm{L}$ & L & ML & VVVL & VL & VVL & $\begin{array}{l}\text { R66 } \\
\text { R68 }\end{array}$ & $\begin{array}{l}\mathrm{VH} \\
\mathrm{vH}\end{array}$ & L & MLL & MLL & $\mathrm{M}$ & VVVL & L & MLL \\
\hline $\begin{array}{l}\text { R67 } \\
\text { R69 }\end{array}$ & $\begin{array}{l}\mathrm{VH} \\
\mathrm{vH}\end{array}$ & $\begin{array}{l}\mathrm{ML} \\
\mathrm{MH}\end{array}$ & $\begin{array}{c}\text { M } \\
\text { MHH }\end{array}$ & $\begin{array}{l}\mathrm{ML} \\
\mathrm{MH}\end{array}$ & $\begin{array}{c}\mathrm{MH} \\
\mathrm{H}\end{array}$ & $\begin{array}{l}\text { VVL } \\
\text { ML }\end{array}$ & $\begin{array}{l}\mathrm{L} \\
\mathrm{M}\end{array}$ & $\begin{array}{l}\mathrm{ML} \\
\mathrm{MH}\end{array}$ & $\begin{array}{l}\text { R668 } \\
\text { R70 }\end{array}$ & $\begin{array}{l}\mathrm{VH} \\
\mathrm{VH}\end{array}$ & $\begin{array}{l}\mathrm{M} \\
\mathrm{H}\end{array}$ & $\mathrm{MH}$ & $\mathrm{MH}$ & $\begin{array}{l}\mathrm{MH} \\
\mathrm{VH}\end{array}$ & VL & MLL & $\begin{array}{c}\mathrm{M} \\
\mathrm{MH}\end{array}$ \\
\hline $\begin{array}{l}609 \\
R 71\end{array}$ & $\begin{array}{l}\mathrm{VH} \\
\mathrm{VH}\end{array}$ & $\begin{array}{l}\mathrm{MH} \\
\mathrm{VH}\end{array}$ & $\begin{array}{l}\mathrm{MHH} \\
\mathrm{VH}\end{array}$ & $\begin{array}{l}\mathrm{MH} \\
\mathrm{vH}\end{array}$ & $\begin{array}{c}\mathrm{H} \\
\mathrm{VVH}\end{array}$ & $\begin{array}{l}\text { ML } \\
\text { MHH }\end{array}$ & $\begin{array}{l}\mathrm{M} \\
\mathrm{H}\end{array}$ & $\begin{array}{l}\mathrm{MH} \\
\mathrm{MHH}\end{array}$ & $\begin{array}{l}R=170 \\
R 72\end{array}$ & $\begin{array}{l}\mathrm{VH} \\
\mathrm{VH}\end{array}$ & $\begin{array}{l}\mathrm{H} \\
\mathrm{VVH}\end{array}$ & $\begin{array}{c}\mathrm{H} \\
\mathrm{VVH}\end{array}$ & $\begin{array}{l}\mathrm{H} \\
\mathrm{VVH}\end{array}$ & $\begin{array}{l}\text { VH } \\
\text { VVVH }\end{array}$ & $\begin{array}{l}\mathrm{M} \\
\mathrm{H}\end{array}$ & $\begin{array}{l}\mathrm{MH} \\
\text { VVVH }\end{array}$ & $\begin{array}{l}\mathrm{MH} \\
\mathrm{MHH}\end{array}$ \\
\hline $\mathrm{R} 73$ & $\mathrm{VVH}$ & $\mathrm{VVL}$ & MLL & MLL & $\mathrm{ML}$ & $\mathrm{EL}$ & $\mathrm{VVL}$ & $\mathrm{EL}$ & R74 & $\mathrm{VVH}$ & $\mathrm{VL}$ & $\mathrm{ML}$ & $\mathrm{ML}$ & M & VVVL & $\mathrm{VL}$ & $\mathrm{vL}$ \\
\hline R75 & $\mathrm{VVH}$ & $\mathrm{L}$ & MH & $\mathrm{M}$ & MH & VVL & MLL & MLL & R76 & VVH & ML & MHH & MHH & $\mathrm{MH}$ & VL & $\mathrm{M}$ & $\mathrm{M}$ \\
\hline $\begin{array}{l}\text { R777 } \\
\text { 707 }\end{array}$ & $\mathrm{VVH}$ & M & H & H & MHH & $\mathrm{L}$ & MHH & MH & $\begin{array}{l}\text { R78 } \\
\text { R78 }\end{array}$ & $\begin{array}{l}\mathrm{VVH} \\
\mathrm{V}\end{array}$ & MH & $\begin{array}{ll}\mathrm{VH} \\
\mathrm{VH}\end{array}$ & $\mathrm{VH}$ & VH & MLL & $\begin{array}{r}\mathrm{H} \\
\text { WV }\end{array}$ & MH \\
\hline $\begin{array}{l}\mathrm{R} / 9 \\
\mathrm{R} 81\end{array}$ & $\begin{array}{l}\text { VVH } \\
\text { VVH }\end{array}$ & $\begin{array}{c}\mathrm{H} \\
\text { VVH }\end{array}$ & EH & $\begin{array}{l}\text { VVH } \\
\text { EH }\end{array}$ & $\begin{array}{l}\text { VVH } \\
\text { EH }\end{array}$ & $\begin{array}{c}\mathrm{M} \\
\mathrm{MHH}\end{array}$ & $\begin{array}{l}\mathrm{VH} \\
\mathrm{EH}\end{array}$ & $\underset{\mathrm{HH}}{\mathrm{MH}}$ & R80 & $\mathrm{VVH}$ & $\mathrm{VH}$ & $\mathrm{VVVH}$ & VVVH & $\mathrm{VVVH}$ & $\mathrm{MH}$ & $\mathrm{VVH}$ & $\mathrm{H}$ \\
\hline
\end{tabular}


Table 5. The rules defined for the CT fuzzy inference system.

\begin{tabular}{|c|c|c|c|c|c|c|c|c|c|c|c|c|c|c|c|c|c|}
\hline & Input & & & & & & & & & Input & & & & $\mathrm{Ou}_{\mathrm{u}}$ & & & \\
\hline Rules & Speed & Depth of Cut & $\begin{array}{c}\text { Tangential } \\
\text { Cutting Force }\end{array}$ & $\begin{array}{c}\text { Radial } \\
\text { Cutting Force }\end{array}$ & Temperature & $\begin{array}{c}\text { Surface } \\
\text { Roughness }\end{array}$ & $\begin{array}{c}\text { Chip } \\
\text { Compression } \\
\text { Ratio }\end{array}$ & Shear Angle & Rules & Speed & Depth of Cut & $\begin{array}{c}\begin{array}{c}\text { Tangential } \\
\text { Cutting Force }\end{array} \\
\end{array}$ & $\begin{array}{c}\text { Radial } \\
\text { Cutting Force } \\
\end{array}$ & Temperature & $\begin{array}{c}\begin{array}{c}\text { Surface } \\
\text { Roughness }\end{array} \\
\end{array}$ & $\begin{array}{c}\text { Chip } \\
\text { Compression } \\
\text { Ratio }\end{array}$ & Shear Angle \\
\hline R1 & $\mathrm{VVL}$ & VVL & VVVL & EL & $\mathrm{EL}$ & $\mathrm{VL}$ & $\mathrm{EL}$ & L & R2 & VVL & $\mathrm{VL}$ & VL & VVL & VVVL & MLL & VVL & ML \\
\hline R3 & VVL & $\mathrm{L}$ & MLL & & VVL & $\mathrm{ML}$ & & MH & R4 & VVL & $\mathrm{ML}$ & M & $\mathrm{ML}$ & VVL & M & MLL & $\mathrm{H}$ \\
\hline $\begin{array}{l}\mathrm{R} 5 \\
\mathrm{R} 7\end{array}$ & $\begin{array}{l}\mathrm{VVL} \\
\text { VVL }\end{array}$ & $\underset{\mathrm{H}}{\mathrm{M}}$ & $\begin{array}{l}\mathrm{MH} \\
\mathrm{VH}\end{array}$ & $\underset{\mathrm{VH}}{\mathrm{M}}$ & $\begin{array}{l}\mathrm{VL} \\
\mathrm{L}\end{array}$ & $\begin{array}{l}\mathrm{MH} \\
\mathrm{VH}\end{array}$ & ML & $\begin{array}{l}\mathrm{VH} \\
\mathrm{VVH}\end{array}$ & $\begin{array}{l}\text { R6 } \\
\text { R8 }\end{array}$ & $\begin{array}{l}\text { VVL } \\
\text { VVL }\end{array}$ & $\begin{array}{l}\mathrm{MH} \\
\mathrm{VH}\end{array}$ & $\begin{array}{l}\mathrm{MHH} \\
\mathrm{VHHH}\end{array}$ & $\begin{array}{l}\text { MHHH } \\
\text { VVVH }\end{array}$ & $\begin{array}{l}\text { VL } \\
\text { MLL }\end{array}$ & $\begin{array}{c}\mathrm{H} \\
\mathrm{VVVH}\end{array}$ & $\begin{array}{l}\mathrm{MH} \\
\mathrm{VH}\end{array}$ & $\begin{array}{l}\text { VH } \\
\text { vVVH }\end{array}$ \\
\hline $\begin{array}{l}\mathrm{R} 7 \\
\mathrm{R} 9\end{array}$ & VVL & $\begin{array}{l}\mathrm{n} \\
\mathrm{VVH}\end{array}$ & $\mathrm{EH}$ & $\mathrm{EH}$ & $\begin{array}{l}\text { MLL } \\
\text { MLL }\end{array}$ & $\mathrm{EH}$ & $\begin{array}{c}\mathrm{H} \\
\mathrm{VVH}\end{array}$ & $\begin{array}{l}\mathrm{VH} \\
\mathrm{EH}\end{array}$ & $\begin{array}{l}\text { R8 } \\
\text { R10 }\end{array}$ & VL & $\begin{array}{l}\text { VV } \\
\text { VVL }\end{array}$ & $\begin{array}{l}\mathrm{VHHH} \\
\mathrm{EL}\end{array}$ & EL & $\begin{array}{l}\text { MLL } \\
\text { VVVL }\end{array}$ & $\begin{array}{l}\text { VVVH } \\
\text { VVL }\end{array}$ & $\begin{array}{l}\mathrm{VH} \\
\mathrm{VVVL}\end{array}$ & $\begin{array}{l}\text { VVVH } \\
\text { MLL }\end{array}$ \\
\hline R11 & $\mathrm{vL}$ & & & & & & VVL & MH & R12 & & $\mathrm{L}$ & ML & $\mathrm{L}$ & VI & ML & VVV & \\
\hline R13 & VL & $\mathrm{ML}$ & $\mathrm{ML}$ & $\mathrm{ML}$ & $\mathrm{VL}$ & $\mathrm{ML}$ & ML & $\mathrm{VH}$ & R14 & VL & $\mathrm{M}$ & MH & $\mathrm{MH}$ & $\mathrm{L}$ & M & M & $\begin{array}{l}\mathrm{H} \\
\mathrm{VVH}\end{array}$ \\
\hline R15 & $\mathrm{VL}$ & $\mathrm{MH}$ & мHH & MHH & $\mathrm{L}$ & MHH & MHH & $\mathrm{VVH}$ & R16 & $\mathrm{ML}$ & $\mathrm{H}$ & $\mathrm{VH}$ & $\mathrm{VH}$ & MLL & ${ }_{\mathrm{H}}^{\mathrm{M}}$ & $\mathrm{H}$ & $\mathrm{VVVH}$ \\
\hline R17 & $\mathrm{VL}$ & VH & VVVH & VVVH & $\mathrm{ML}$ & $\mathrm{VVH}$ & $\mathrm{VH}$ & VVVH & R18 & $\mathrm{VL}$ & VVH & $\mathrm{EH}$ & $\mathrm{EH}$ & $\mathrm{ML}$ & vVVH & $\mathrm{VVH}$ & $\mathrm{EH}$ \\
\hline $\begin{array}{l}\text { R19 } \\
\text { R21 }\end{array}$ & L & $\mathrm{VVL}$ & $\begin{array}{l}\mathrm{VVVL} \\
\mathrm{VL}\end{array}$ & $\begin{array}{l}\text { VVVL } \\
\text { MLL }\end{array}$ & VVL & $\mathrm{VVL}$ & L & $\stackrel{\mathrm{L}}{\mathrm{L}}$ & $\begin{array}{l}\text { R20 } \\
\text { R22 }\end{array}$ & $\mathrm{L}$ & $\begin{array}{l}\mathrm{VL} \\
\mathrm{ML}\end{array}$ & $\mathrm{VVL}$ & VL & $\mathrm{VL}$ & $\begin{array}{l}\mathrm{VL} \\
\mathrm{ML}\end{array}$ & MLL & L \\
\hline $\begin{array}{l}\text { R21 } \\
\text { R23 }\end{array}$ & ${ }_{\mathrm{L}}^{\mathrm{L}}$ & $\stackrel{\mathrm{L}}{\mathrm{M}}$ & $\begin{array}{l}\mathrm{VL} \\
\mathrm{M}\end{array}$ & $\begin{array}{l}\text { MLL } \\
\text { MH }\end{array}$ & $\begin{array}{l}\text { VL } \\
\text { MLL }\end{array}$ & $\begin{array}{l}\mathrm{L} \\
\mathrm{M}\end{array}$ & $\begin{array}{l}\mathrm{ML} \\
\mathrm{M}\end{array}$ & $\mathrm{MLL}$ & 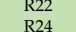 & ${ }_{\mathrm{L}}^{\mathrm{L}}$ & $\begin{array}{l}\mathrm{ML} \\
\mathrm{MH}\end{array}$ & $\begin{array}{l}\text { ML } \\
\text { MHH }\end{array}$ & $\begin{array}{l}\text { M } \\
\text { MHн }\end{array}$ & $\stackrel{\mathrm{L}}{\mathrm{M}}$ & ML & $\begin{array}{l}\mathrm{ML} \\
\mathrm{MHH}\end{array}$ & $\begin{array}{l}\mathrm{ML} \\
\mathrm{MH}\end{array}$ \\
\hline R25 & $\begin{array}{l}\mathrm{L} \\
\mathrm{L}\end{array}$ & $\begin{array}{l}\mathrm{M} \\
\mathrm{H}\end{array}$ & $\begin{array}{l}\mathrm{M} \\
\mathrm{H}\end{array}$ & ${ }_{\mathrm{VH}}^{\mathrm{MH}}$ & MH & $\begin{array}{l}\mathrm{M} \\
\mathrm{H}\end{array}$ & ${ }_{\mathrm{H}}^{\mathrm{M}}$ & $\begin{array}{l}\text { MH } \\
\text { MH }\end{array}$ & $\begin{array}{l}\text { R24 } \\
\text { R26 }\end{array}$ & $\begin{array}{l}\mathrm{L} \\
\mathrm{L}\end{array}$ & $\begin{array}{l}\mathrm{MH} \\
\mathrm{VH}\end{array}$ & VH & VVH & $\begin{array}{l}\text { M } \\
\text { MHH }\end{array}$ & VH & VH & ${ }_{\mathrm{H}}^{\mathrm{MH}}$ \\
\hline R27 & $\mathrm{L}$ & $\mathrm{VVH}$ & $\mathrm{VVH}$ & $\mathrm{VVVH}$ & $\mathrm{H}$ & vVH & vVH & $\mathrm{VH}$ & $\mathrm{R} 28$ & $\mathrm{ML}$ & VVL & VVVL & VVVL & VVL & vVL & $\mathrm{ML}$ & $\mathrm{L}$ \\
\hline R29 & $\mathrm{ML}$ & $\mathrm{VL}$ & VVL & $\mathrm{VL}$ & VL & $\mathrm{VL}$ & ML & $\mathrm{L}$ & R30 & $\mathrm{ML}$ & $\mathrm{L}$ & VL & $\mathrm{L}$ & L & $\mathrm{L}$ & ML & MLL \\
\hline $\begin{array}{l}{ }_{\mathrm{R}}^{231} 31 \\
\mathrm{R} 3\end{array}$ & $\begin{array}{l}\mathrm{ML} \\
\mathrm{ML}\end{array}$ & $\begin{array}{l}\mathrm{ML} \\
\mathrm{MH}\end{array}$ & $\begin{array}{l}\mathrm{ML} \\
\mathrm{MHH}\end{array}$ & $\begin{array}{l}\text { ML } \\
\text { MHH }\end{array}$ & MLL & ML & $\mathrm{M}$ & $\mathrm{ML}$ & $\begin{array}{l}\text { R32 } \\
\text { R34 }\end{array}$ & $\mathrm{ML}$ & $\mathrm{M}$ & $\mathrm{M}$ & M & $\mathrm{ML}$ & $\mathrm{M}$ & $\mathrm{M}$ & M \\
\hline $\begin{array}{l}\text { R33 } \\
\text { R35 }\end{array}$ & $\begin{array}{l}\mathrm{ML} \\
\mathrm{ML}\end{array}$ & $\begin{array}{l}\mathrm{MH} \\
\mathrm{vH}\end{array}$ & $\begin{array}{c}\mathrm{MHH} \\
\mathrm{vH}\end{array}$ & $\begin{array}{l}\mathrm{MHH} \\
\mathrm{VHH}\end{array}$ & $\begin{array}{l}\text { M } \\
\text { MHН }\end{array}$ & $\begin{array}{l}\text { MHH } \\
\mathrm{v} V \mathrm{H}\end{array}$ & $\begin{array}{l}\mathrm{MH} \\
\mathrm{vH}\end{array}$ & $\begin{array}{c}\mathrm{MH} \\
\mathrm{H}\end{array}$ & $\begin{array}{l}\text { R34 } \\
\text { R36 }\end{array}$ & $\begin{array}{l}\mathrm{ML} \\
\mathrm{ML}\end{array}$ & $\begin{array}{c}\mathrm{H} \\
\text { VyH }\end{array}$ & $\begin{array}{c}\mathrm{H} \\
\mathrm{VVH}\end{array}$ & $\begin{array}{c}\mathrm{H} \\
\text { vyvH }\end{array}$ & $\begin{array}{c}\mathrm{MH} \\
\mathrm{H}\end{array}$ & $\begin{array}{c}\mathrm{H} \\
\text { vvVH }\end{array}$ & $\begin{array}{l}\mathrm{MHH} \\
\mathrm{V} \mathrm{VH}\end{array}$ & $\begin{array}{c}\mathrm{MHH} \\
\mathrm{vH}\end{array}$ \\
\hline $\begin{array}{l}\text { R35 } \\
\text { R37 }\end{array}$ & M & $\begin{array}{l}\mathrm{VH} \\
\mathrm{VVL}\end{array}$ & $\begin{array}{l}\mathrm{VH} \\
\mathrm{VVVL}\end{array}$ & $\begin{array}{l}\text { VVVI } \\
\text { VVH }\end{array}$ & $\begin{array}{l}\mathrm{MHH} \\
\mathrm{VL}\end{array}$ & $\mathrm{VL}$ & $\begin{array}{l}\mathrm{VH} \\
\mathrm{M}\end{array}$ & $\begin{array}{l}\mathrm{H} \\
\mathrm{VVL}\end{array}$ & $\begin{array}{l}\text { r56 } \\
\text { R38 }\end{array}$ & $\begin{array}{l}\mathrm{ML} \\
\mathrm{M}\end{array}$ & $\mathrm{VL}$ & $\mathrm{VL}$ & $\mathrm{VL}$ & $\begin{array}{l}\mathrm{H} \\
\mathrm{L}\end{array}$ & L & M & $\begin{array}{c}\mathrm{VH} \\
\mathrm{L}\end{array}$ \\
\hline $\begin{array}{l}\text { R39 } \\
\text { R3 }\end{array}$ & $\mathrm{M}$ & L & L & MLL & MLL & MLL & $\mathrm{MH}$ & ML & R40 & M & $\mathrm{ML}$ & $\mathrm{ML}$ & $\mathrm{M}$ & $\mathrm{ML}$ & ML & MH & $\mathrm{M}$ \\
\hline R41 & M & $\mathrm{M}$ & $\mathrm{M}$ & $\mathrm{MH}$ & M & M & MH & MHH & R42 & $\mathrm{M}$ & MH & MHH & MH & MH & MH & MHH & MHH \\
\hline $\begin{array}{l}\text { R43 } \\
\text { R45 }\end{array}$ & M & $\mathrm{H}$ & VH & $\begin{array}{l}\mathrm{H} \\
\mathrm{yyyzH}\end{array}$ & MHH & MHH & $\begin{array}{l}\mathrm{VH} \\
\mathrm{y}\end{array}$ & $\begin{array}{l}\mathrm{H} \\
\mathrm{H}\end{array}$ & $\begin{array}{l}\text { R44 } \\
\text { R }\end{array}$ & $\mathrm{M}$ & VH & $\mathrm{VVH}$ & VVH & $\mathrm{H}$ & $\mathrm{VH}$ & VVH & $\begin{array}{l}\text { VH } \\
\text { VyyYH }\end{array}$ \\
\hline 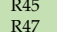 & $\begin{array}{c}\mathrm{M} \\
\mathrm{MH}\end{array}$ & $\mathrm{VVH}$ & $\begin{array}{l}\mathrm{VVH} \\
\mathrm{VVYH}\end{array}$ & VVVH & VH & VVH & VVVH & $\mathrm{VVH}$ & $\begin{array}{l}\text { R46 } \\
\text { R48 }\end{array}$ & $\mathrm{MH}$ & VVL & EL & EL & $\begin{array}{l}\mathrm{L} \\
\mathrm{M}\end{array}$ & $\mathrm{L}$ & $\mathrm{MH}$ & VVVL \\
\hline $\begin{array}{l}\text { R47 } \\
\text { R49 }\end{array}$ & $\begin{array}{l}\mathrm{MH} \\
\mathrm{MH}\end{array}$ & $\begin{array}{l}\mathrm{VL} \\
\mathrm{ML}\end{array}$ & $\mathrm{ML}$ & $\mathrm{ML}$ & $\begin{array}{c}\mathrm{MLL} \\
\mathrm{M}\end{array}$ & $\begin{array}{l}\mathrm{ML} \\
\mathrm{MH}\end{array}$ & $\begin{array}{l}\text { MH } \\
\text { MHH }\end{array}$ & $\begin{array}{l}\mathrm{VL} \\
\mathrm{M}\end{array}$ & $\begin{array}{l}\text { R48 } \\
\text { R50 }\end{array}$ & $\begin{array}{l}\mathrm{MH} \\
\mathrm{MH}\end{array}$ & $\begin{array}{l}\mathrm{L} \\
\mathrm{M}\end{array}$ & $\begin{array}{l}\mathrm{L} \\
\mathrm{M}\end{array}$ & M & $\begin{array}{l}\mathrm{ML} \\
\mathrm{MH}\end{array}$ & $\begin{array}{l}\text { M } \\
\text { MHH }\end{array}$ & $\begin{array}{l}\text { MHH } \\
\text { MHH }\end{array}$ & $\begin{array}{l}\mathrm{MLL} \\
\mathrm{MH}\end{array}$ \\
\hline R51 & MH & $\mathrm{MH}$ & $\mathrm{MH}$ & $\mathrm{MH}$ & $\mathrm{MH}$ & $\mathrm{H}$ & $\mathrm{H}$ & MHH & R52 & MH & $\mathrm{H}$ & $\mathrm{vH}$ & ${ }_{\mathrm{H}}^{\mathrm{H}}$ & $\mathrm{H}$ & $\mathrm{vH}$ & $\mathrm{vH}$ & $\mathrm{H}$ \\
\hline R53 & MH & $\mathrm{VH}$ & VVH & VVH & $\mathrm{VH}$ & VVH & VVH & H & R54 & MH & VVH & VVVH & VVVH & $\mathrm{VH}$ & VVVH & VVVH & VH \\
\hline $\begin{array}{l}\text { R55 } \\
\text { R55 }\end{array}$ & $\mathrm{H}$ & VVL & EL & EL & MLL & VVL & M & VVL & $\begin{array}{l}\text { R56 } \\
\text { R58 }\end{array}$ & $\mathrm{H}$ & VL & VVVL & $\begin{array}{l}\mathrm{VL} \\
\mathrm{ML}\end{array}$ & ML & VL & $\mathrm{M}$ & $\mathrm{L}$ \\
\hline $\begin{array}{l}\text { R57 } \\
\text { R59 }\end{array}$ & $\begin{array}{l}\mathrm{H} \\
\mathrm{H}\end{array}$ & $\begin{array}{l}\mathrm{L} \\
\mathrm{M}\end{array}$ & ${ }_{\mathrm{M}}^{\mathrm{L}}$ & $\begin{array}{l}\mathrm{L} \\
\mathrm{M}\end{array}$ & $\begin{array}{l}\text { M } \\
\text { MHН }\end{array}$ & $\begin{array}{l}\mathrm{L} \\
\mathrm{ML}\end{array}$ & $\begin{array}{l}\mathrm{MH} \\
\mathrm{MH}\end{array}$ & $\begin{array}{l}\mathrm{ML} \\
\mathrm{MH}\end{array}$ & 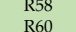 & $\begin{array}{l}\mathrm{H} \\
\mathrm{H}\end{array}$ & $\begin{array}{l}\mathrm{ML} \\
\mathrm{MH}\end{array}$ & $\begin{array}{l}\mathrm{ML} \\
\mathrm{MHH}\end{array}$ & $\begin{array}{l}\mathrm{ML} \\
\mathrm{MHH}\end{array}$ & $\mathrm{MHH}$ & $\begin{array}{l}\text { MLL } \\
\text { ML }\end{array}$ & $\begin{array}{l}\mathrm{MH} \\
\text { MH }\end{array}$ & $\begin{array}{l}\text { M } \\
\text { MHH }\end{array}$ \\
\hline R61 & $\begin{array}{l}\mathrm{H} \\
\mathrm{H}\end{array}$ & $\begin{array}{l}\mathrm{M} \\
\mathrm{H}\end{array}$ & $\begin{array}{l}\mathrm{M} \\
\mathrm{H}\end{array}$ & $\begin{array}{l}\mathrm{M} \\
\mathrm{H}\end{array}$ & $\begin{array}{l}\mathrm{MHH} \\
\mathrm{vH}\end{array}$ & $\begin{array}{l}\mathrm{ML} \\
\mathrm{M}\end{array}$ & $\begin{array}{c}\mathrm{MH} \\
\mathrm{H}\end{array}$ & $\begin{array}{c}\mathrm{MH} \\
\mathrm{H}\end{array}$ & $\begin{array}{l}\text { R60 } \\
\text { R62 }\end{array}$ & $\begin{array}{l}\mathrm{H} \\
\mathrm{H}\end{array}$ & $\begin{array}{l}\mathrm{MH} \\
\mathrm{VH}\end{array}$ & $\begin{array}{l}\mathrm{M} \text { MHH } \\
\mathrm{VVH}\end{array}$ & VVH & $\begin{array}{l}\mathrm{H} \\
\mathrm{VVH}\end{array}$ & $\begin{array}{l}\mathrm{ML} \\
\mathrm{MH}\end{array}$ & $\begin{array}{l}\mathrm{MHH} \\
\mathrm{VH}\end{array}$ & $\mathrm{VH}$ \\
\hline R63 & $\mathrm{H}$ & $\mathrm{vVH}$ & VVVH & VVVH & $\mathrm{VVH}$ & $\mathrm{MH}$ & $\mathrm{VVH}$ & $\mathrm{vVH}$ & R64 & VH & VVL & VVVL & $\mathrm{EL}$ & MLL & $\mathrm{VVVL}$ & MH & $\mathrm{VVVL}$ \\
\hline R65 & $\mathrm{VH}$ & VL & VL & VVL & M & VVL & MH & $\mathrm{VL}$ & R66 & VH & L & $\mathrm{L}$ & $\mathrm{L}$ & MH & $\mathrm{VL}$ & MH & $\mathrm{L}$ \\
\hline $\begin{array}{l}\text { R67 } \\
\text { P69 }\end{array}$ & $\mathrm{VH}$ & $\mathrm{ML}$ & ML & ML & MHH & L & MHH & $\mathrm{ML}$ & $\begin{array}{l}\text { R68 } \\
\text { R70 }\end{array}$ & $\mathrm{VH}$ & $\mathrm{M}$ & $\mathrm{M}$ & $\mathrm{ML}$ & $\mathrm{H}$ & L & MHH & M \\
\hline $\begin{array}{l}\text { R69 } \\
\text { R71 }\end{array}$ & $\mathrm{VH}$ & MH & MHH & MH & VH & MLL & $\begin{array}{c}\mathrm{H} \\
\mathrm{V}\end{array}$ & $\mathrm{MH}$ & $\begin{array}{l}\text { R70 } \\
\text { R72 }\end{array}$ & VH & H & H & H & VVH & ML & VH & MHH \\
\hline $\begin{array}{l}\text { R7/ } \\
\text { R73 }\end{array}$ & $\begin{array}{l}\mathrm{VH} \\
\mathrm{VVH}\end{array}$ & $\begin{array}{l}\mathrm{VH} \\
\mathrm{VVL}\end{array}$ & $\begin{array}{l}\mathrm{VH} \\
\mathrm{EL}\end{array}$ & $\begin{array}{l}\mathrm{VVH} \\
\mathrm{EL}\end{array}$ & ML & $\begin{array}{l}\mathrm{M} \\
\mathrm{EL}\end{array}$ & MHH & $\begin{array}{l}\mathrm{H} \\
\mathrm{EL}\end{array}$ & 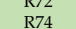 & $\begin{array}{l}\mathrm{VH} \\
\mathrm{VVH}\end{array}$ & $\begin{array}{l}\mathrm{VWH} \\
\mathrm{VL}\end{array}$ & $\mathrm{VVL}$ & $\begin{array}{l}\text { EH } \\
\text { VVL }\end{array}$ & M & $\begin{array}{l}\mathrm{M} \\
\mathrm{VVVL}\end{array}$ & $\mathrm{MHH}$ & $\begin{array}{l}\mathrm{VH} \\
\mathrm{VVL}\end{array}$ \\
\hline R75 & $\mathrm{VVH}$ & $\mathrm{L}$ & $\mathrm{L}$ & $\mathrm{L}$ & MHH & VVL & $\mathrm{H}$ & $\mathrm{L}$ & R76 & $\mathrm{VVH}$ & $\mathrm{ML}$ & MLL & $\mathrm{ML}$ & $\mathrm{H}$ & VL & $\mathrm{H}$ & MLL \\
\hline R77 & VVH & $\mathrm{M}$ & $\mathrm{ML}$ & ML & $\mathrm{VH}$ & VL & $\mathrm{H}$ & $\mathrm{ML}$ & R78 & VVH & MH & MH & MH & VH & L & VH & M \\
\hline $\begin{array}{l}\text { R79 } \\
\text { R81 }\end{array}$ & $\begin{array}{l}\text { VVH } \\
\text { vyH }\end{array}$ & $\begin{array}{c}\mathrm{H} \\
\mathrm{yyH}\end{array}$ & $\begin{array}{c}\mathrm{H} \\
\mathrm{yyH}\end{array}$ & $\begin{array}{c}\mathrm{H} \\
\mathrm{FH}\end{array}$ & $\begin{array}{l}\mathrm{VVH} \\
\mathrm{EH}\end{array}$ & MLL & $\begin{array}{l}\mathrm{VVH} \\
\mathrm{EH}\end{array}$ & MH & R80 & $\mathrm{VVH}$ & $\mathrm{VH}$ & $\mathrm{VH}$ & VVH & VVVH & M & VVVH & MHH \\
\hline
\end{tabular}




\section{Experimental Work}

A Ti-6Al-7Zr-3Nb-4Mo-0.9Nd designated as a T-6734-0.9Nd rod that had a length of $90 \mathrm{~m}$ and a diameter of $25 \mathrm{~mm}$ was received from the Technical University of Braunschweig, Germany. A thermosetting adhesive was used to join the received small piece of Ti-alloy to a mild steel specimen to be able to mount it in the chuck of a Lathe machine. DNMG 150608 MF1 CP500 inserts were used in the experiments, and details can be found elsewhere [3,7].

Three cutting speeds of 10,20, and $30 \mathrm{~m} / \mathrm{min}$ and three DoC levels $(100,200$, and $300 \mu \mathrm{m})$ were selected for the experiments to validate the predicted simulation results. Each set of experiments was repeated three times, and no cutting fluid was used in the tests.

A modified universal Harrison M-300 Lathe machine was used to conduct the experiments on the as received alloys for the CT and UVAT process. The cutting forces were measured using a force sensor (KIAG-SWISS/Type9257A) made by Kistler. The axial force component was ignored during experimentation due to its low intensity compared to $F t$ and $F r$. The frequency and amplitude were set to $20 \mathrm{kHz}$ and $10 \mathrm{um}$, respectively, in the UVAT process. A calibrated FLIR (ThermaCAMTM SC-3000) thermal system was used for the measurement of the maximum process-zone temperature in $\mathrm{CT}$ and UVAT. The quick view specialized software was used for the analysis of the results.

The surface quality assessments of the machined samples were conducted on a Zygo ${ }^{\circledR}$ newview-5000 interferometer. The $R a$ was assessed at the tested cutting conditions, and data were taken at various locations. The chips produced at various cutting conditions were collected and analyzed for CCR and SA in UVAT and CT. The chip collected at $100 \mu \mathrm{m}$ and higher speeds were hard to mount in Bakelite resin and were excluded from experimentation. Additionally, the metallographic analysis of the alloy is not included in the current work.

\section{Results and Discussion}

\subsection{Simulations Results}

The commercially available simulation tool MATLAB 2014b was used for the developed algorithms based on the fuzzy logic inference system for UVAT and CT. The developed models were able to predict $F r$, Ft, process zone temperature, surface roughness, CCR, and $\mathrm{SA}$ at various speed and depth-of-cut (DoCs) combinations as in input parameters.

The predicted $F t$ and $F r$ at selected cutting speeds and DoCs in CT and UVAT are presented in Figures 5 and 6, respectively. The models predicted the cutting forces at various cutting parameters based on the rules defined and are consistent with the previously published work on UVAT and CT processes [3,10,38,42]. The model developed for UVAT process predicted a lower average value of $F t$ and $F r$ at various combinations of speed and DoCs when compared to the CT results.

A gradual increase in the predicted $F t$ and $F r$ was observed, with an increase in the speed and DoC during the UVAT process, as expected. Similarly, the model developed for $\mathrm{CT}$ foretold a gradual increase in the cutting forces, with a rise in the DoC. However, a minor variation in the $F t$ and $F r$ at selected cutting speeds was achieved in CT and was in good agreement with the findings of Muhammad et al. [3,7]. An average decline of approximately $60-70 \%$ in the cutting forces was described by the simulation of the UVAT process when compared to $\mathrm{CT}$ at lower tested cutting speeds.

The maximum temperature predicted in the UVAT and CT processes by the developed models is shown in Figure 7. A gradual increase in the maximum temperature with the cutting speed and DoC was measured in during the UVAT and CT processes [10,42]. However, the temperature level predicted in UVAT was slightly higher when compared to the temperature level predicted for $\mathrm{CT}$ due to the additional increase in the relative velocity due to vibration coupling on the cutting insert, which is in good agreement with Naseer et al. [43]. 


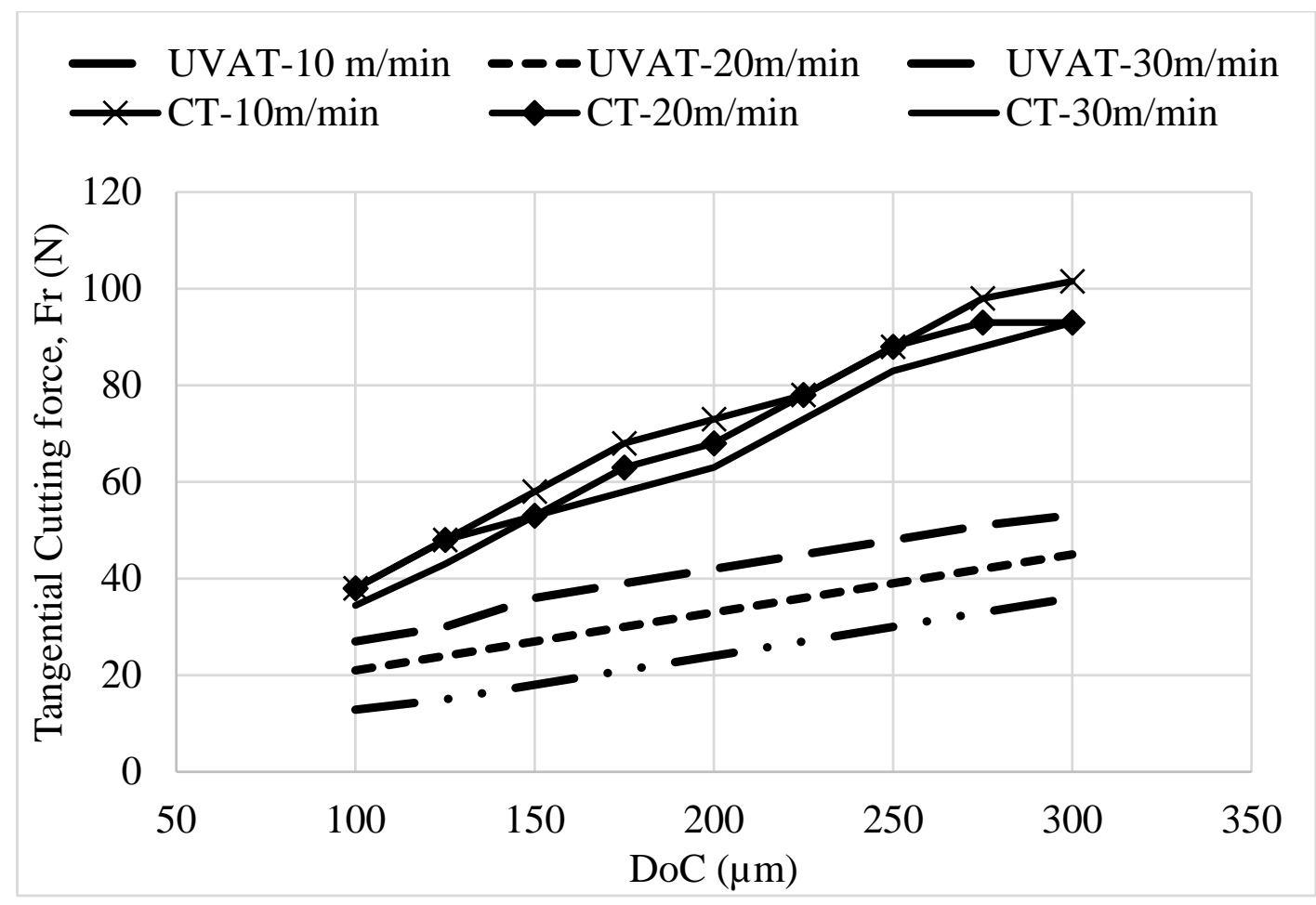

Figure 5. The predicted Ft in CT and UVAT.

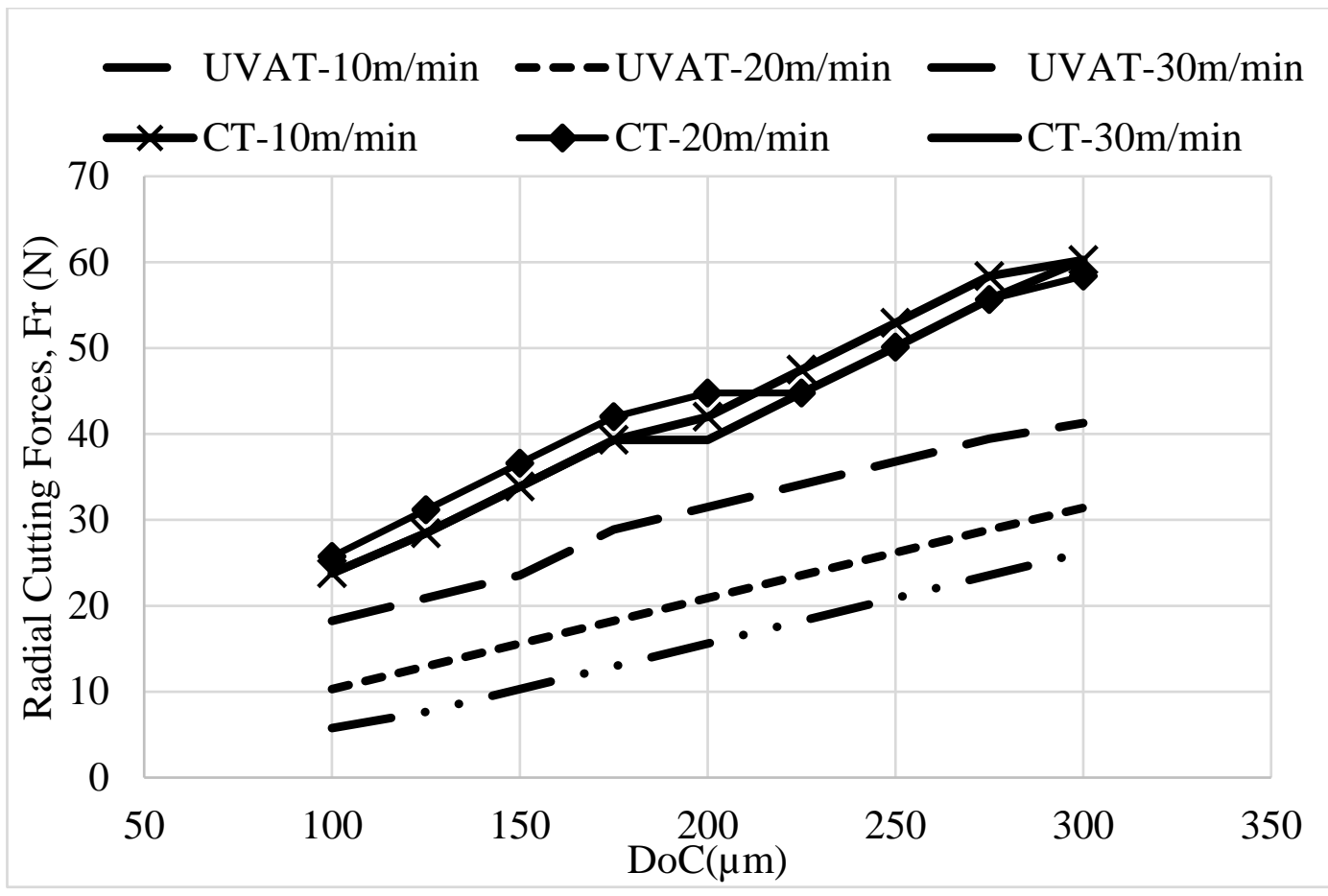

Figure 6. The predicted $F r$ in CT and UVAT. 


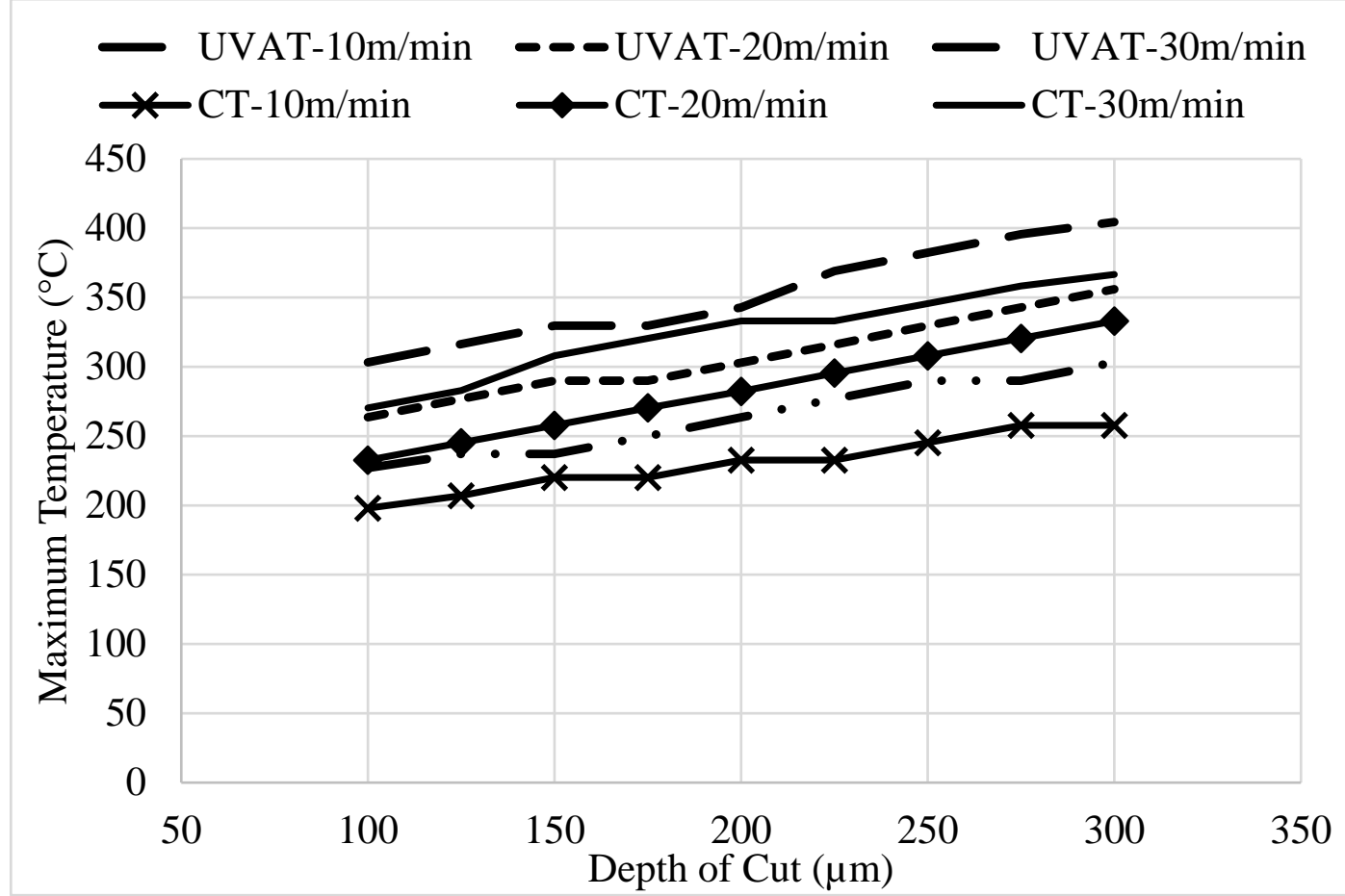

Figure 7. The predicted maximum temperature results in UVAT and CT.

The developed simulation models were utilized to predict the surface roughness parameter $(R a)$ for the tested combinations of speed and DoC, as demonstrated in Figure 8 . The model predicted a significant improvement in $R a$ due to the ironing effect of the cutting insert on the machine surface. Similarly, the models developed for the UVAT and CT processes predicted an improvement in the surface quality with a growth in cutting speed, which is in good agreement with work of Silberschmidt et al. [44].

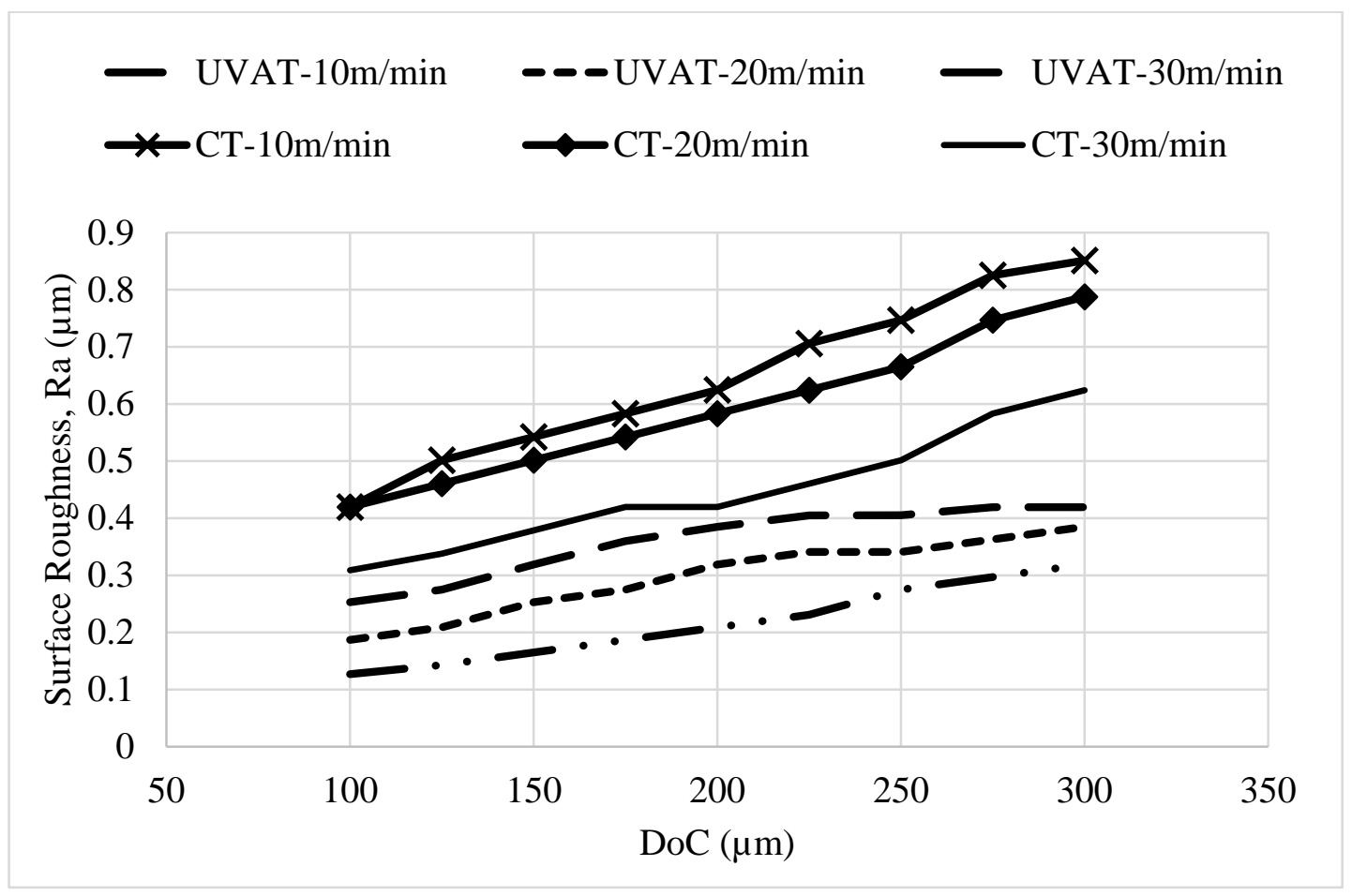

Figure 8. The predicted $R a$ in UVAT and CT. 
The developed model was also used to predict the CCR and SA of the formed chips at various speed and DoC combinations. The predicted levels of CRR and SA are presented in Figures 9 and 10, respectively. The models predicted a gradual increase in the CCR, showing growth in the speed and DoC in the studied processes [45]. However, the CCR in UVAT is significantly higher when matched to the CCR in CT.

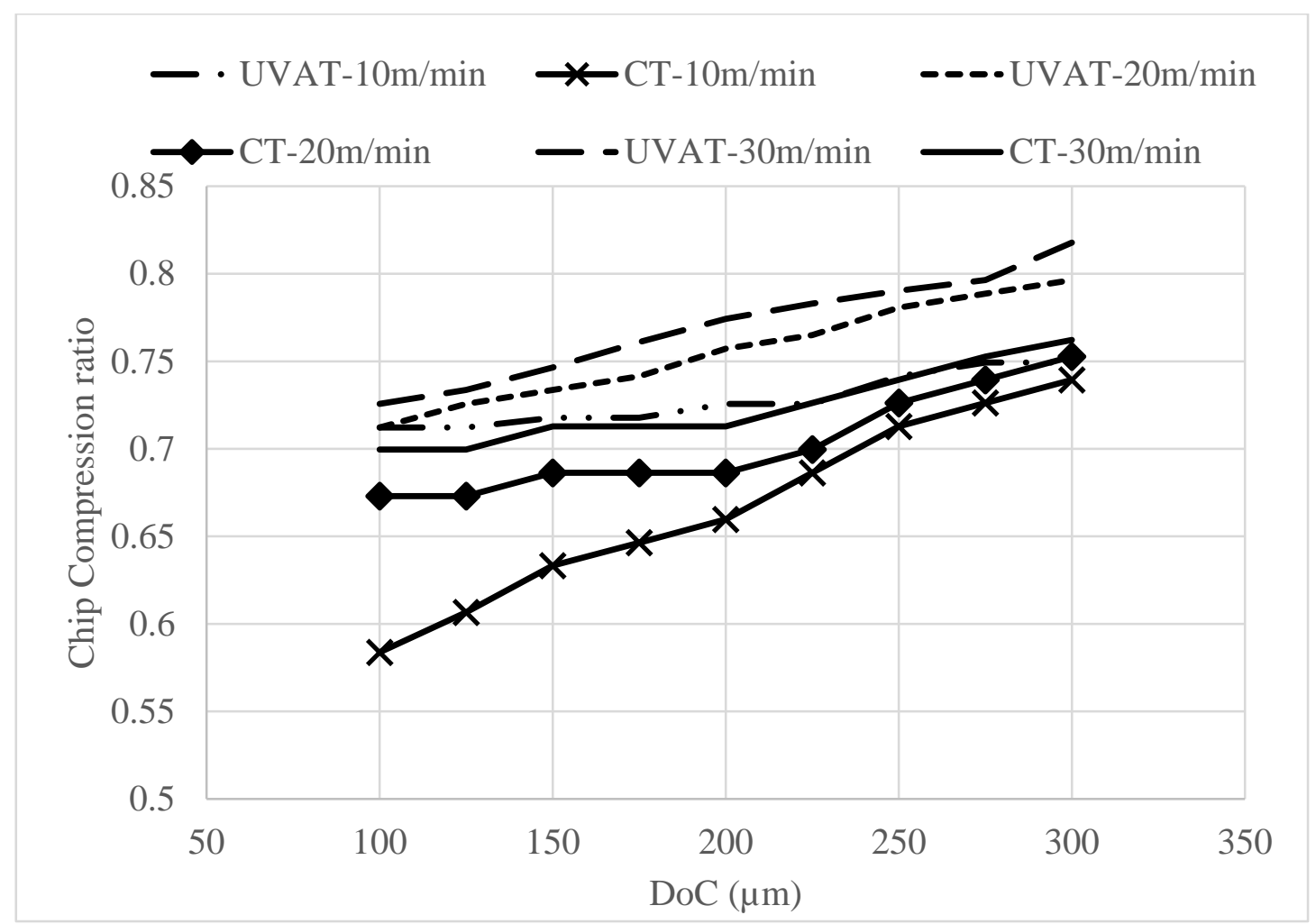

Figure 9. Predicted level of CCR in UVAT and CT.

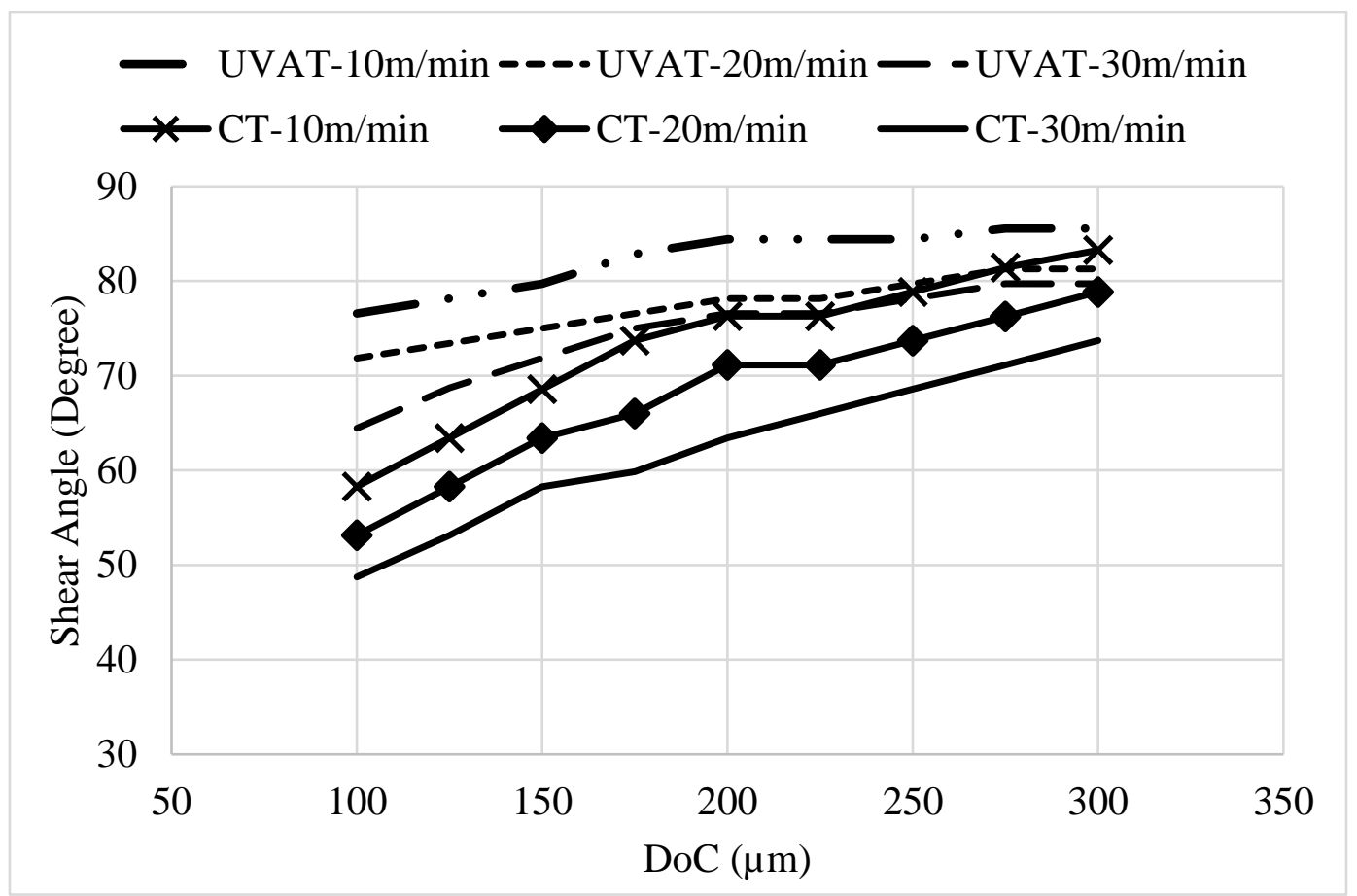

Figure 10. The simulated SA variation with speed and DoC in UVAT and CT. 
A gradual rise in the SA was predicted in the CT and UVAT processes with DoC. However, with a growth in speed, a slight drop in the shear angle was noticed, which is in good agreement with [45].

\subsection{Experimental Results}

The experimental results demonstrate a significant decline of approximately $60-70 \%$ in the cutting forces in UVAT, as shown in Figure 11. At lower cutting speed of $10 \mathrm{~m} / \mathrm{min}$, the levels of $F t$ observed in CT at 100, 200, and $300 \mu \mathrm{m}$ DoCs were 38, 72, and $101 \mathrm{~N}$, respectively. Similarly, 23, 43, and $61 \mathrm{~N}$ forces in the radial direction were recorded for the same cutting conditions. On the other hand, a significantly lower level of $F t$ was measured in the UVAT process. The effect of vibrations on cutting forces is substantial at lower cutting speeds due to longer separation between the insert edge and chip [17].

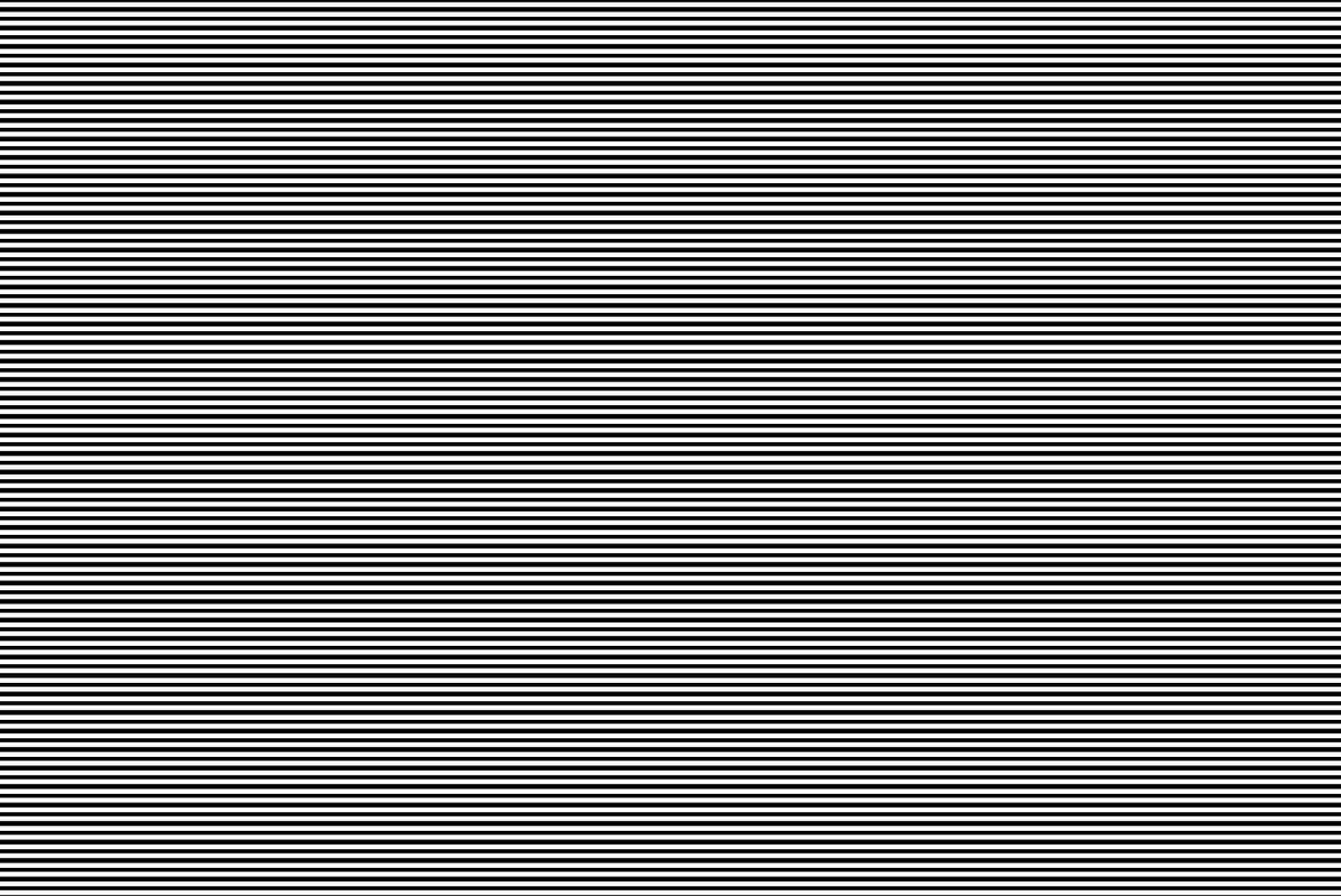

Figure 11. Cutting forces at various DoCs and constant speed of $10 \mathrm{~m} / \mathrm{min}$.

The cutting forces were measured at various cutting speeds in CT, and it was found that speed has no significant effect on it (see Figure 12). However, in the UVAT process, a gradual increase in $F t$ and $F r$ was recorded with an increase in cutting speed. The $F t$ merely increased from 38 to $54 \mathrm{~N}$ when the speed was increased from 10 to $30 \mathrm{~m} / \mathrm{min}$ and $\mathrm{DoC}=300 \mu \mathrm{m}$. The same trend was also seen at lower DoCs. 


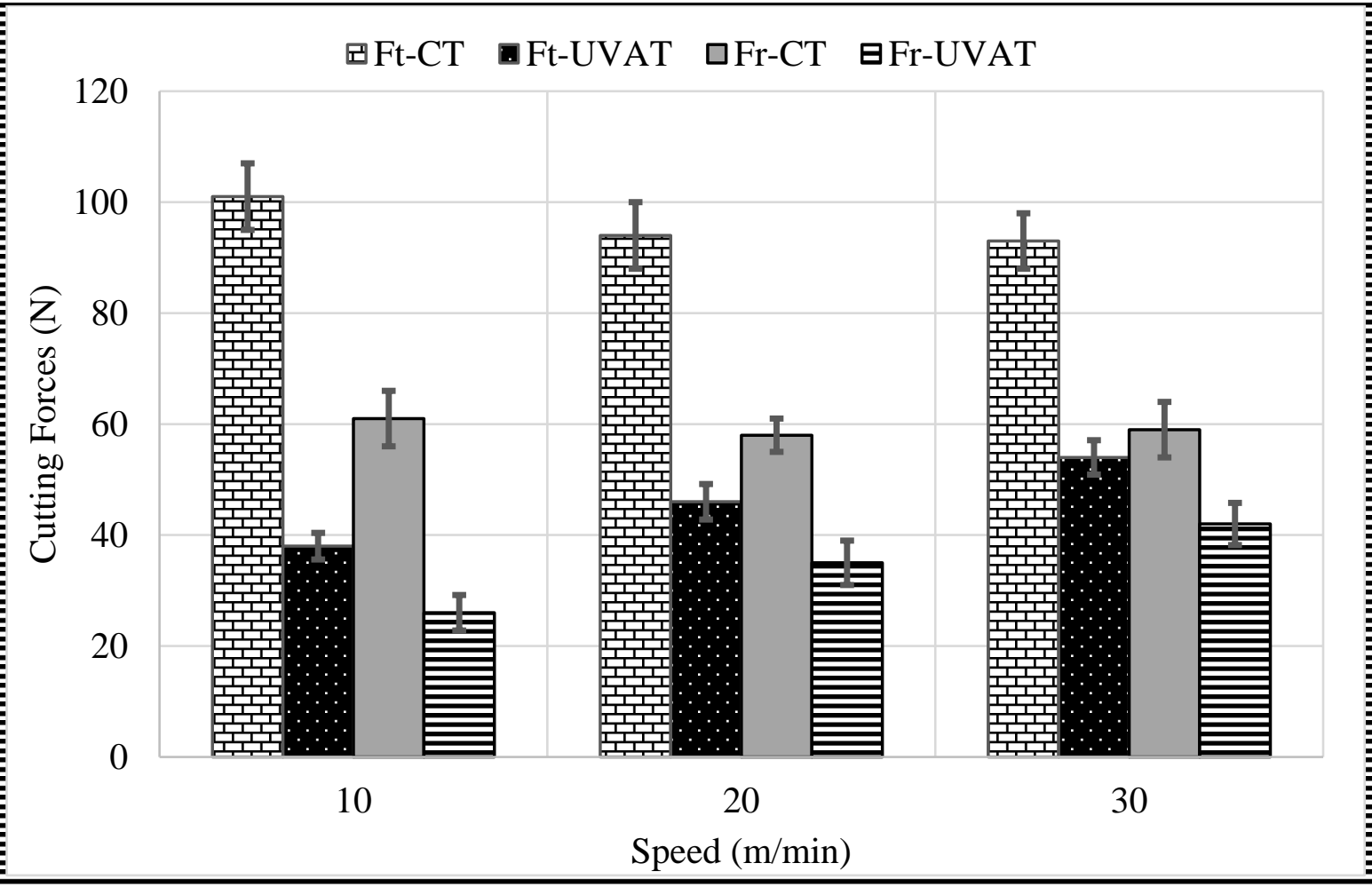

Figure 12. Experimentally measured cutting forces at various speeds and $\mathrm{DoC}=300 \mu \mathrm{m}$.

The maximum temperatures recorded during the CT and UVAT processes are shown in Figure 13. A slightly higher temperature level was recorded in UVAT $\left(408{ }^{\circ} \mathrm{C}\right)$ when matched to CT $\left(327^{\circ} \mathrm{C}\right)$ at the $30 \mathrm{~m} / \mathrm{min}$ cutting speed and the $300 \mu \mathrm{m}$ DoC. The same trend was observed in all of the tested cutting conditions. The increase in temperature can be linked with escalation in the relative velocity of the tool in one complete vibrational cycle and with the additional power provided to the cutting edge in the form of vibration $[7,42,43]$. The same trend was observed when the DoC was increased as well; however, the impact of speed is foremost, as expected [39,40,46].

The quality of the product is assessed through many factors, but one of the most prominent factors is the surface topography of a machined part. A substantial enhancement in the machine surface quality was achieved in the UVAT process when judged with CT in the same cutting conditions as those presented in Figure 14. The $R a$ value measured at $30 \mathrm{~m} / \mathrm{min}$ in CT was still higher when compared to that obtained during the UVAT process at $10 \mathrm{~m} / \mathrm{min}$. The tool movement in one complete vibration cycle produced an ironing effect on the turned surface, and a reduction of approximately $36-50 \%$ in the $R a$ was achieved at all of the tested conditions in the UVAT process when matched to CT. 


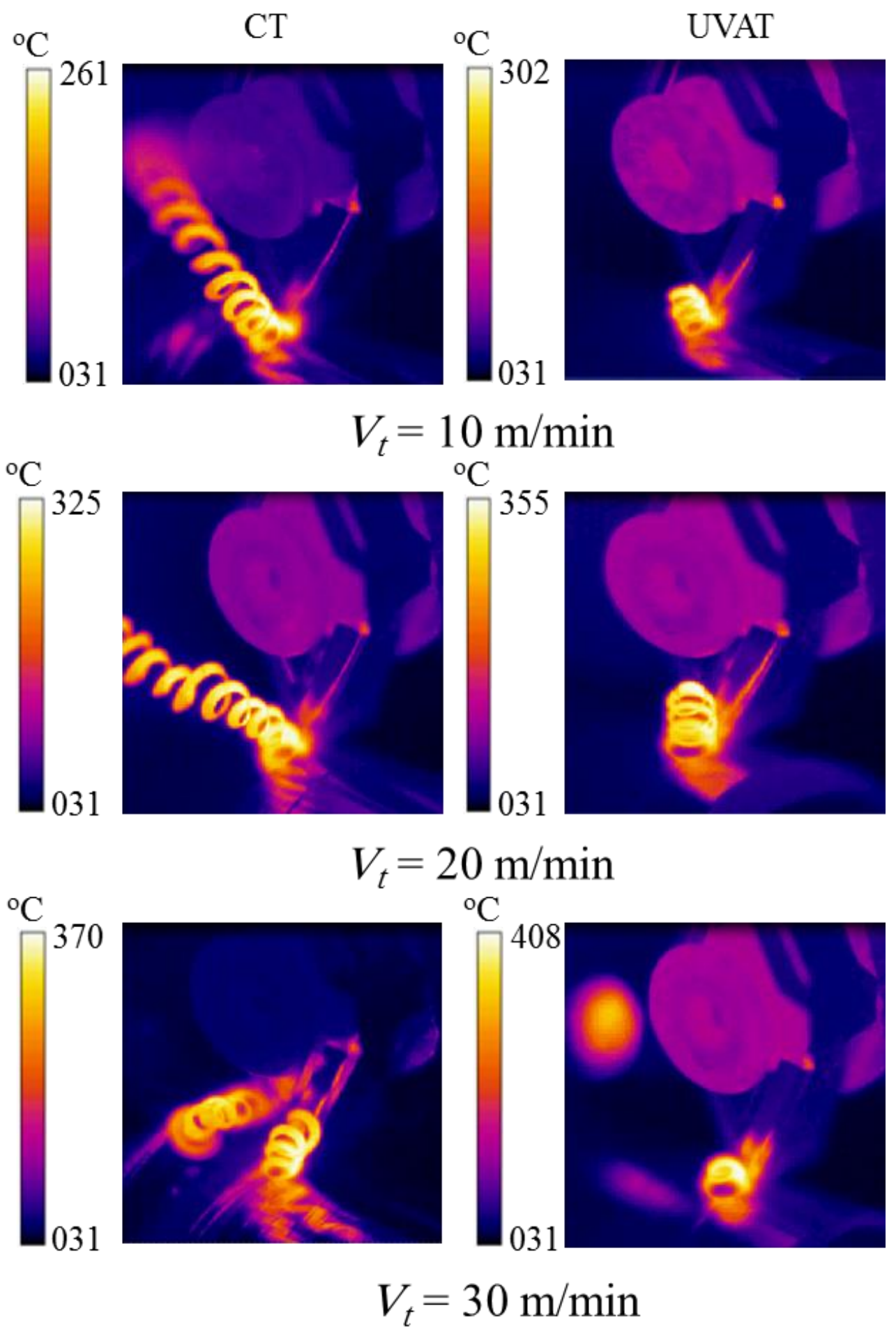

Figure 13. Measured temperature in CT and UVAT.

The studied alloys resulted in discontinuous chips in the CT and UVAT processes in all of the tested conditions. The chips were analyzed for CRR and SA calculations. The chip size produced during UVAT was substantially smaller when matched to the chip produced during CT in the same cutting conditions. The discontinuity of the chips resulted from the addition of $\mathrm{Nd}$, which has a lower melting temperature. The chip samples from the UVAT and CT processes are presented in Figure 15. 
(CT)

(UVAT)

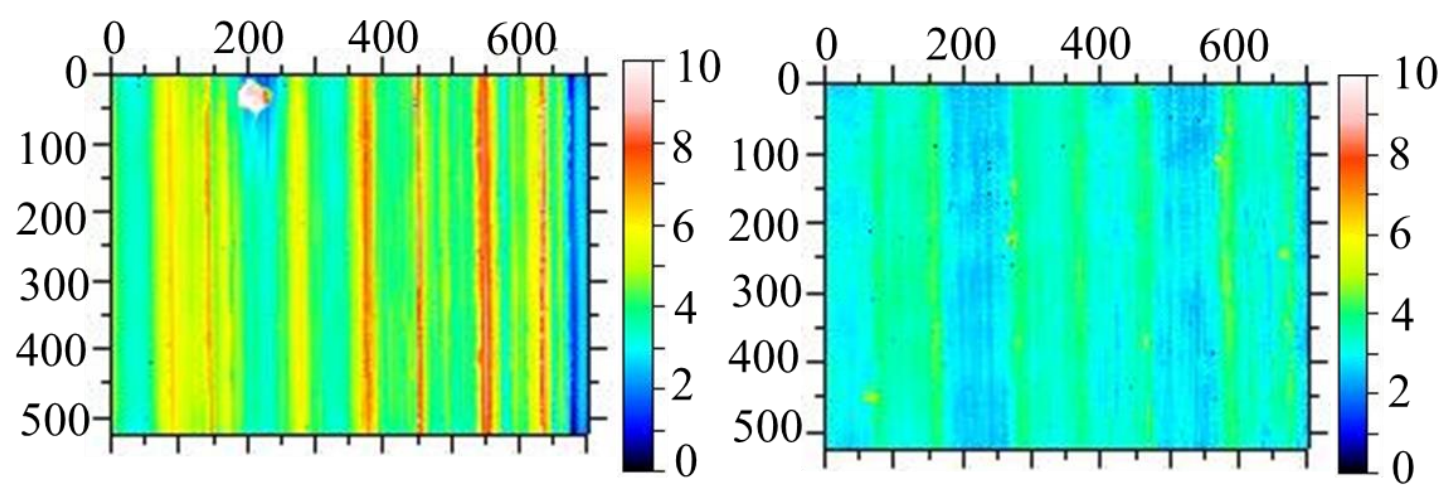

$\mathrm{DoC}=300 \mu \mathrm{m}$ and cutting speed of $10 \mathrm{~m} / \mathrm{min}$

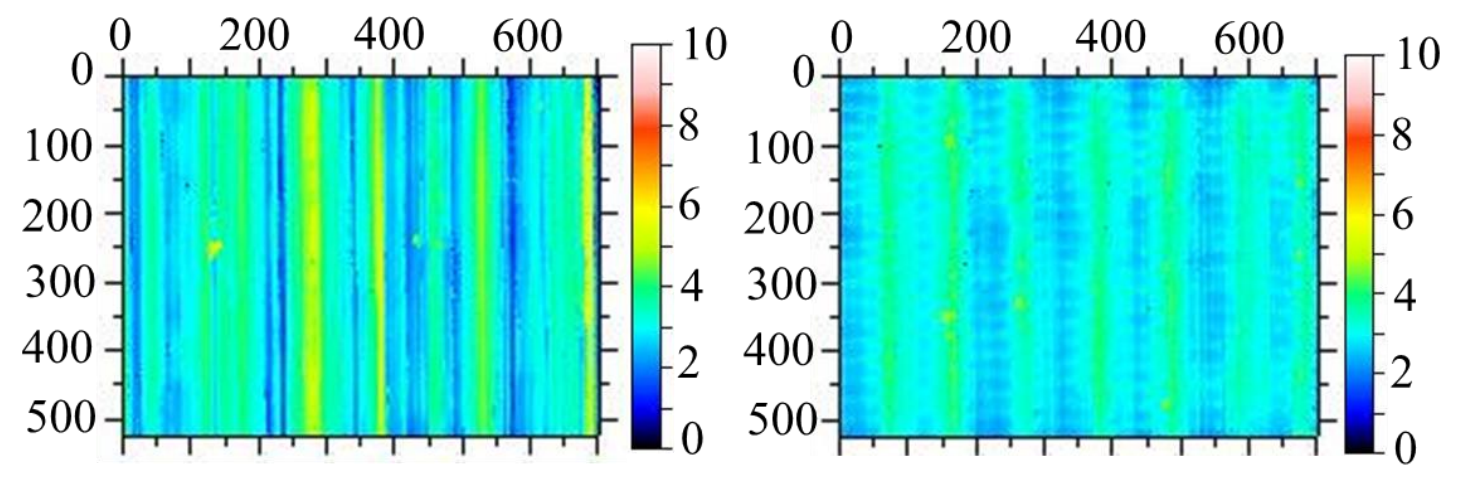

$\mathrm{DoC}=300 \mu \mathrm{m}$ and cutting speed of $30 \mathrm{~m} / \mathrm{min}$

Figure 14. Optical scans of machined surfaces.

\section{(CT)}

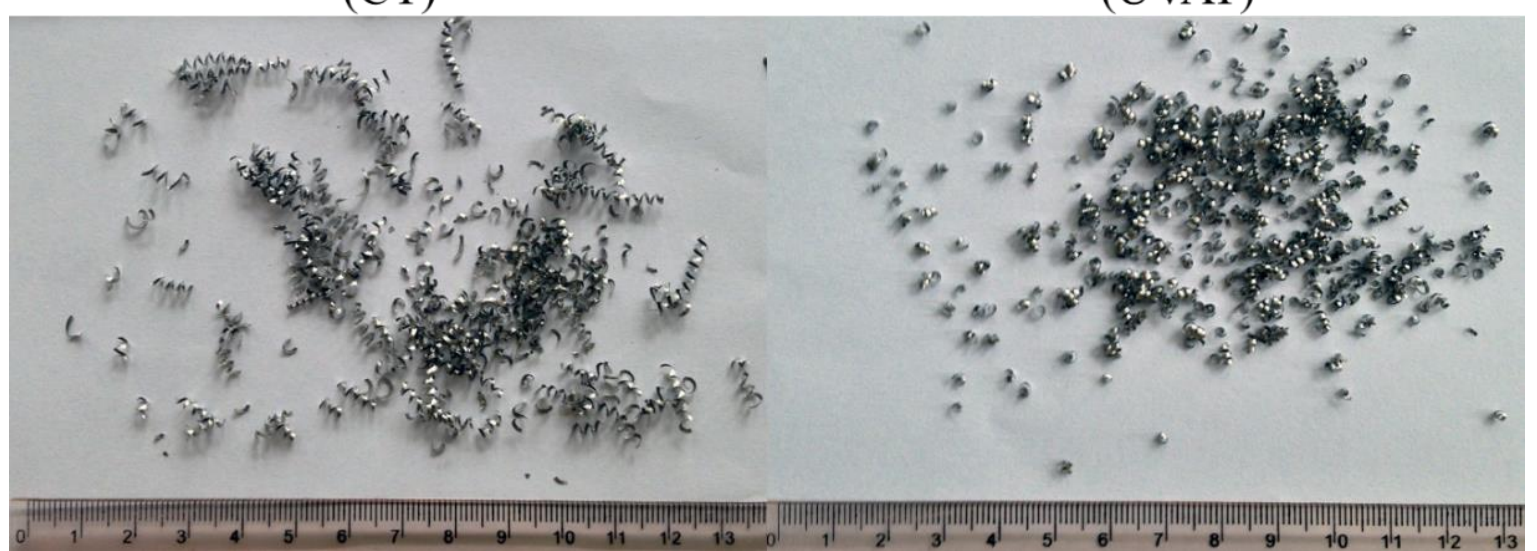

Figure 15. Chips of the studied alloy collected from the UVAT and CT processes.

The CCR was calculated using Equation (2), and SA was calculated using Equation (3) for the CT and UVAT processes [47].

$$
\begin{gathered}
\mathrm{CCR}=\frac{H_{\text {max }}+H_{\min }}{2 d_{c}} . \\
\mathrm{SA}=\operatorname{Tan}^{-1}\left(\frac{r \cos \alpha}{1-r \sin \alpha}\right)
\end{gathered}
$$




$$
r=\frac{t_{f}}{t_{o}}
$$

where $H_{\text {max }}$ is the maximum height measured in serrated chips, the measured minimum height is denoted by $H_{\text {min }}$, the net chip thickness is represented by $d_{c}, \alpha$ is the rake angle of the cutting insert, which is $14.6^{\circ}$ for the CP-500 inserts, $t_{f}$ is the thickness of the chips after machining, and $t_{o}$ is the initial chip thickness before machining.

The calculated CCR in the UVAT process was slightly higher than that of CT, as presented in Figure 16, and the possible reason for this is the vibration imposed on the cutting insert. A minor increase in the CCR was observed with an increase in cthe utting speed during both CT and UVAT, as expected

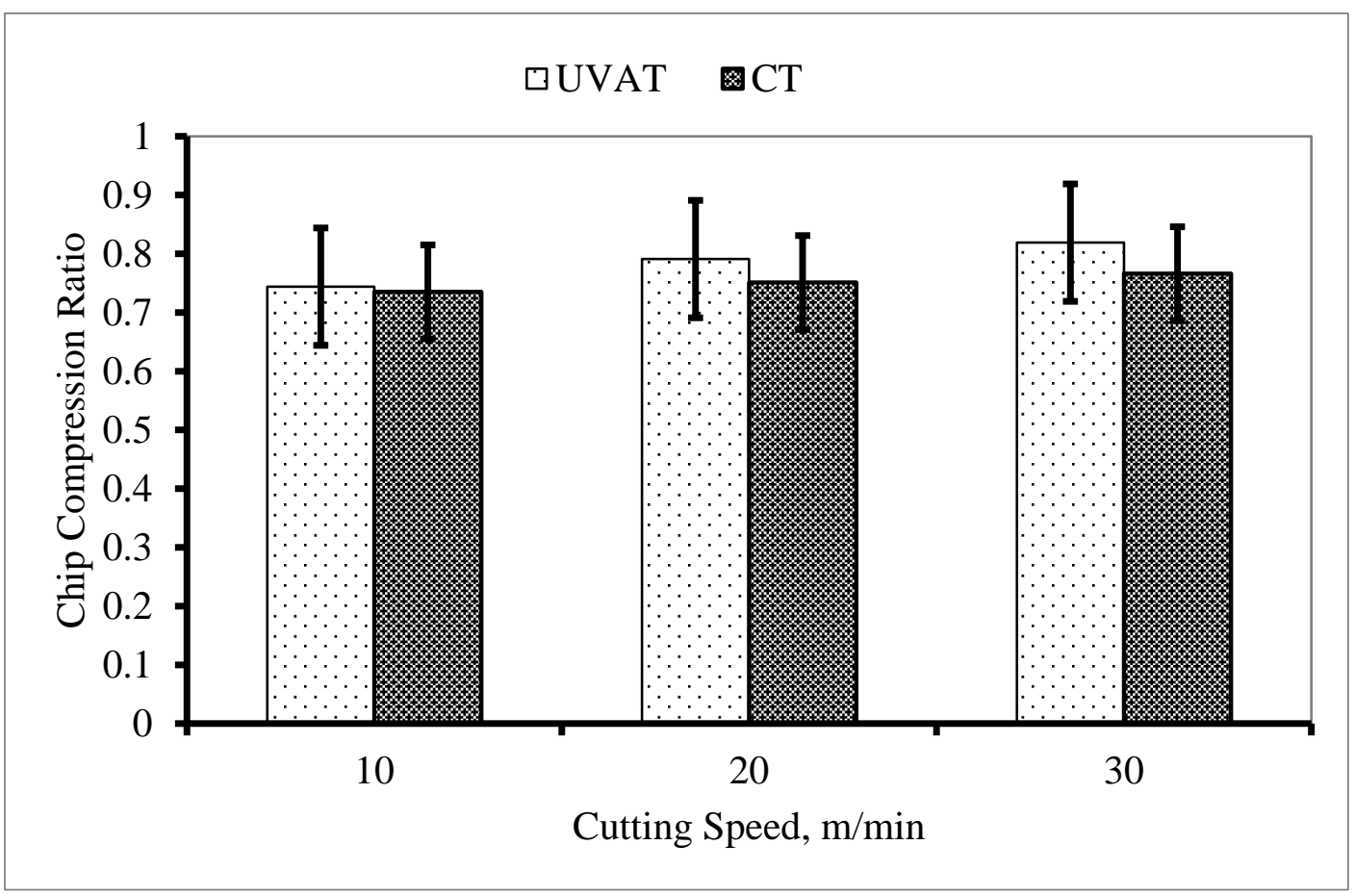

Figure 16. The calculated CCR at $\mathrm{DoC}=300 \mu \mathrm{m}$.

Similarly, the calculated SA for the CT and UVAT processes is shown in Figure 17. The results for the UVAT process showed a relatively higher magnitude of SA when compared to that of CT. An average SA of $86.2^{\circ}, 81.2^{\circ}$, and $78.2^{\circ}$ was measured at 10,20 , and $30 \mathrm{~m} / \mathrm{min}$, respectively, whereas in $\mathrm{CT}$, the calculated SA was $84.8^{\circ}, 78.9^{\circ}$ and $73.6^{\circ}$, correspondingly. The increase of SA in the UVAT process can be attributed to the intermittent contact between the cutting insert and the chip. The vibro-impact phenomena resulted in more plastic deformation at the process zone when compared to CT. Additionally, the higher temperature level in the UVAT process also expedited the plastic deformation of the shear zone and resulted in a slight increase in SA. A minor decline in SA was noted with an increase in the cutting speed in the CT and UVAT processes. 


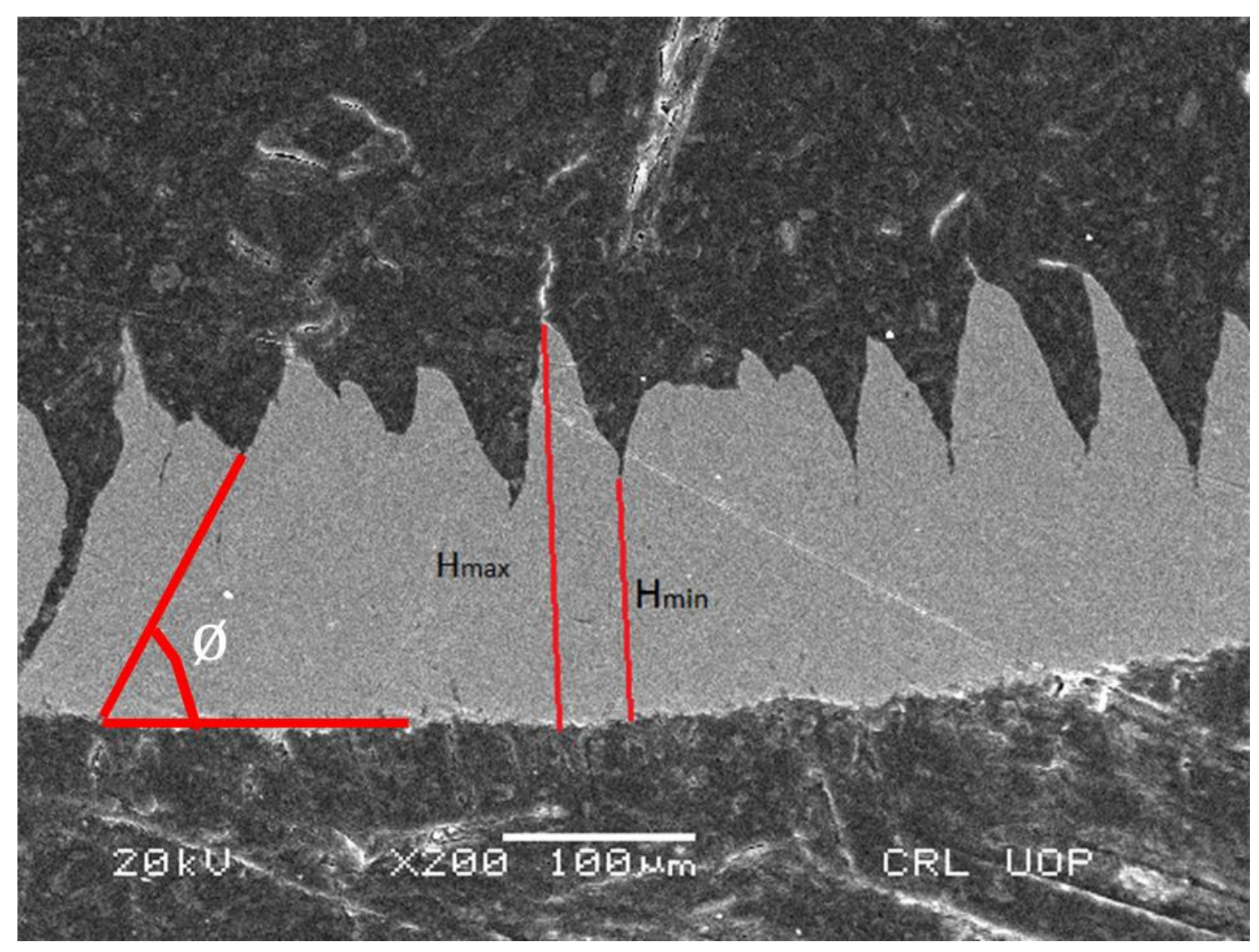

Figure 17. SEM image of the analyzed chip.

\subsection{Comparison of Simulated and Experimental Results}

The data obtained from simulation of the developed algorithm are consistent with the experimental results for both of the studied processes. A comparative analysis of the simulation and experimental results is depicted in Tables 6 and 7 for CT and UVAT, respectively. The predicted $F t$ and $F r$ in CT at $10 \mathrm{~m} / \mathrm{min}$ velocity and $\mathrm{DoC}=300 \mu \mathrm{m}$ are $101.55 \mathrm{~N}$ and $60.25 \mathrm{~N}$, respectively, whereas the tests led to an average value of approximately $101 \mathrm{~N}$ and $61 \mathrm{~N}$, which are consistent with the simulation results. Similarly, the cutting forces predicted by the developed model for $\mathrm{CT}$ at all of the tested conditions are in good agreement with the experimental results (see Table 6).

Moreover, at $\mathrm{DoC}=300 \mu \mathrm{m}$, the predicted maximum process zone temperature in $\mathrm{CT}$ was $257.74{ }^{\circ} \mathrm{C}$ at $10 \mathrm{~m} / \mathrm{min}$ speed (see Table 6). The model predicted temperature levels of $333.00{ }^{\circ} \mathrm{C}$ and $366.66^{\circ} \mathrm{C}$ when the speed was increased to $20 \mathrm{~m} / \mathrm{min}$ and $30 \mathrm{~m} / \mathrm{min}$, respectively. The experimental results showed temperatures of $262.00{ }^{\circ} \mathrm{C}, 325.00{ }^{\circ} \mathrm{C}$, and $370.00{ }^{\circ} \mathrm{C}$, correspondingly. The model predicted that the process zone temperature would have a maximum and minimum error of $3.11 \%$ and $0.71 \%$, respectively, as shown in Figure 6.

Furthermore, the developed fuzzy model for CT predicted CCR values of 0.5838 , 0.6464 , and 0.7394 at $10 \mathrm{~m} / \mathrm{min}$ speed studied DoC. The experimental results were measured, and the results showed that the model predicted the CCR with an accuracy of $99 \%$. The simulations and experimental results of CCR are presented in Table 6. 
Table 6. Comparative analysis of simulation and experimental results in CT.

\begin{tabular}{|c|c|c|c|c|c|c|c|c|c|c|c|c|c|}
\hline \multicolumn{2}{|c|}{ Cutting Conditions } & \multicolumn{6}{|c|}{ Simulation Results } & \multicolumn{6}{|c|}{ Experimental Results } \\
\hline $\begin{array}{l}\text { Speed } \\
(\mathrm{m} / \mathrm{min})\end{array}$ & $\begin{array}{l}\text { DOC } \\
(\mu \mathrm{m})\end{array}$ & $\begin{array}{c}F t \\
(\mathbf{N})\end{array}$ & $\begin{array}{l}F r \\
(\mathrm{~N})\end{array}$ & $\begin{array}{l}\mathrm{T}_{\max } \\
\left({ }^{\circ} \mathrm{C}\right)\end{array}$ & $\begin{array}{c}R a \\
(\mu \mathrm{m})\end{array}$ & CCR & $\begin{array}{l}\text { SA } \\
\left({ }^{\circ}\right)\end{array}$ & $\begin{array}{l}F t \\
(\mathrm{~N})\end{array}$ & $\begin{array}{l}F r \\
(\mathrm{~N})\end{array}$ & $\begin{array}{l}\mathrm{T}_{\max } \\
\left({ }^{\circ} \mathrm{C}\right)\end{array}$ & $\begin{array}{c}R a \\
(\mu \mathrm{m})\end{array}$ & CCR & $\begin{array}{l}\text { SA } \\
\left({ }^{\circ}\right)\end{array}$ \\
\hline 10 & 100 & 38.00 & 23.78 & 198.00 & 0.419 & 0.5838 & 58.2 & $38 \pm 5$ & $23 \pm 4$ & 195 & $0.413 \pm 0.036$ & $0.58 \pm 0.15$ & $58.59 \pm 10$ \\
\hline 10 & 200 & 68.00 & 39.32 & 220.14 & 0.583 & 0.6464 & 73.7 & $72 \pm 7$ & $43 \pm 3$ & 227 & $0.621 \pm 0.038$ & $0.65 \pm 0.20$ & $77.56 \pm 08$ \\
\hline 10 & 300 & 101.55 & 60.25 & 257.74 & 0.851 & 0.7394 & 83.3 & $101 \pm 6$ & $61 \pm 5$ & 262 & $0.861 \pm 0.050$ & $0.735 \pm 0.08$ & $84.88 \pm 07$ \\
\hline 20 & 100 & 38.00 & 25.72 & 232.66 & 0.419 & 0.6730 & 53.1 & $35 \pm 4$ & $24 \pm 3$ & 231 & $0.401 \pm 0.050$ & - & - \\
\hline 20 & 200 & 63.00 & 42.00 & 270.34 & 0.542 & 0.6860 & 66.0 & $68 \pm 5$ & $45 \pm 2$ & 276 & $0.601 \pm 0.040$ & $0.68 \pm 0.20$ & $71.67 \pm 09$ \\
\hline 20 & 300 & 93.00 & 58.38 & 333.00 & 0.787 & 0.7530 & 78.8 & $94 \pm 6$ & $58 \pm 3$ & 325 & $0.783 \pm 0.050$ & $0.75 \pm 0.10$ & $78.89 \pm 06$ \\
\hline 30 & 100 & 34.44 & 23.78 & 270.34 & 0.308 & 0.6996 & 48.7 & $34 \pm 3$ & $23 \pm 3$ & 269 & $0.297 \pm 0.060$ & - & - \\
\hline 30 & 200 & 63.00 & 39.32 & 333.00 & 0.419 & 0.7128 & 63.4 & $64 \pm 3$ & $40 \pm 4$ & 326 & $0.419 \pm 0.047$ & $0.71 \pm 0.17$ & $63.46 \pm 12$ \\
\hline 30 & 300 & 93.00 & 60.25 & 366.60 & 0.624 & 0.7622 & 73.7 & $93 \pm 5$ & $59 \pm 5$ & 370 & $0.611 \pm 0.046$ & $0.77 \pm 0.10$ & $73.67 \pm 05$ \\
\hline
\end{tabular}

Table 7. Comparative analysis of experimental and simulation results in UVAT.

\begin{tabular}{|c|c|c|c|c|c|c|c|c|c|c|c|c|c|}
\hline \multicolumn{2}{|c|}{ Cutting Conditions } & \multicolumn{6}{|c|}{ Simulation Results } & \multicolumn{6}{|c|}{ Experimental Results } \\
\hline $\begin{array}{l}\text { Speed } \\
(\mathrm{m} / \mathrm{min})\end{array}$ & $\begin{array}{l}\text { DOC } \\
(\mu \mathrm{m})\end{array}$ & $\begin{array}{c}F t \\
(\mathrm{~N})\end{array}$ & $\begin{array}{l}F r \\
(\mathrm{~N})\end{array}$ & $\begin{array}{l}\mathrm{T}_{\max } \\
\left({ }^{\circ} \mathrm{C}\right)\end{array}$ & $\begin{array}{c}R a \\
(\mu \mathrm{m})\end{array}$ & CCR & $\begin{array}{l}\text { SA } \\
\left({ }^{\circ}\right)\end{array}$ & $\begin{array}{c}F t \\
(\mathrm{~N})\end{array}$ & $\begin{array}{l}F r \\
(\mathrm{~N})\end{array}$ & $\begin{array}{l}\mathrm{T}_{\max } \\
\left({ }^{\circ} \mathrm{C}\right)\end{array}$ & $\begin{array}{c}R a \\
(\mu \mathrm{m})\end{array}$ & CCR & $\begin{array}{l}\text { SA } \\
\left({ }^{\circ}\right)\end{array}$ \\
\hline 10 & 100 & 12.8 & 5.76 & 227.0 & 0.253 & 0.712 & 76.57 & $12 \pm 3.7$ & $05 \pm 3.0$ & 224 & $0.263 \pm 0.02$ & $0.65 \pm 0.16$ & $76.98 \pm 8$ \\
\hline 10 & 200 & 24.0 & 15.60 & 263.5 & 0.385 & 0.724 & 84.42 & $23 \pm 2.6$ & $14 \pm 4.0$ & 261 & $0.381 \pm 0.01$ & $0.72 \pm 0.15$ & $85.46 \pm 3$ \\
\hline 10 & 300 & 36.0 & 26.20 & 303.2 & 0.424 & 0.749 & 85.54 & $38 \pm 2.4$ & $26 \pm 3.2$ & 302 & $0.424 \pm 0.02$ & $0.74 \pm 0.10$ & $86.20 \pm 4$ \\
\hline 20 & 100 & 21.0 & 10.30 & 263.6 & 0.188 & 0.712 & 71.85 & $20 \pm 1.7$ & $10 \pm 3.0$ & 261 & $0.195 \pm 0.02$ & - & - \\
\hline 20 & 200 & 33.0 & 20.89 & 303.0 & 0.319 & 0.753 & 78.10 & $33 \pm 2.2$ & $21 \pm 2.0$ & 303 & $0.313 \pm 0.01$ & $0.68 \pm 0.15$ & $78.30 \pm 6$ \\
\hline 20 & 300 & 45.0 & 31.40 & 355.9 & 0.385 & 0.796 & 81.28 & $46 \pm 3.2$ & $35 \pm 4.0$ & 355 & $0.392 \pm 0.02$ & $0.79 \pm 0.14$ & $81.20 \pm 6$ \\
\hline 30 & 100 & 27.0 & 18.24 & 303.2 & 0.127 & 0.725 & 64.50 & $28 \pm 4.2$ & $18 \pm 3.2$ & 298 & $0.121 \pm 0.02$ & - & - \\
\hline 30 & 200 & 42.0 & 31.50 & 342.8 & 0.209 & 0.774 & 76.60 & $41 \pm 2.3$ & $32 \pm 2.0$ & 340 & $0.219 \pm 0.02$ & $0.77 \pm 0.12$ & $76.70 \pm 4$ \\
\hline 30 & 300 & 53.1 & 41.27 & 404.4 & 0.319 & 0.818 & 79.70 & $54 \pm 3.1$ & $42 \pm 3.8$ & 408 & $0.327 \pm 0.02$ & $0.82 \pm 0.18$ & $78.50 \pm 6$ \\
\hline
\end{tabular}


The predicted and measured $R a$ value of the machined surface using CT is presented in Table 6. The fuzzy model for CT predicted the $R a$ value with a maximum error of $10 \%$, which is still in the acceptable range. The developed model is a good alternative to predict the machine surface quality in CT. Furthermore, the SA predicted by the model is also in good agreement with the calculated results, and corresponding maximum and minimum errors of $8.5 \%$ and $0.05 \%$ were noted.

The developed fuzzy model for the UVAT process was used to predict the cutting forces, maximum process zone temperature, surface roughness, CCR, and SA, as presented in Table 7. At $10 \mathrm{~m} / \mathrm{min}$ speed, the predicted $\mathrm{Ft}$ at 100, 200, and $300 \mu \mathrm{m}$ DoCs were $12.8 \mathrm{~N}$, $24.0 \mathrm{~N}$, and $36.0 \mathrm{~N}$, respectively. The experimental validation demonstrated an error of $6.25 \%, 4.16 \%$, and $5.55 \%$, correspondingly. Similarly, when the speed was increased, a gradual increase in the cutting forces was observed in UVAT both experimentally and through the fuzzy model. However, the overall error was less than $10 \%$ in the predicted forces and showed the acceptability of the developed model for cutting force estimations.

The predicted $\mathrm{Fr}$ by the simulations at all of the tested conditions awere5.76, 15.60, $26.20,10.30,20.89,31.40,18.24,31.50$, and $41.27 \mathrm{~N}$, as presented in Table 7 , whereas the measured $F r$ were 5, 14, 26, 10, 21, 35, 18, 32, and 42 N, correspondingly, and were shown to be in good agreement with the force level predicted by the fuzzy model.

The model was used for the prediction of the maximum process zone temperature during the UVAT process. The model predicted the temperature of the process zone at all of the tested cutting conditions, with a maximum difference of approximately $5{ }^{\circ} \mathrm{C}$ (Max $1.7 \%$ error) and are in good agreement with the experimental results.

The predicted $R a$ of $0.253 \mu \mathrm{m}$ was obtained from the simulation model at $10 \mathrm{~m} / \mathrm{min}$ speed and $100 \mu \mathrm{m}$ DoC. A gradual increase of $0.132 \mu \mathrm{m}$ and $0.171 \mu \mathrm{m}$ in $R a$ was obtained at $200 \mu \mathrm{m}$ and $300 \mu \mathrm{m}$ DoCs, respectively, when compared to Ra achieved at $100 \mu \mathrm{m}$, and kept the speed intact, whereas the experiments resulted in $R a$ of $0.263 \mu \mathrm{m}, 0.381 \mu \mathrm{m}$, and $0.424 \mu \mathrm{m}$, correspondingly. In addition, the fuzzy model is sensitive to cutting speed and showed surface roughness improvements with speed. The model also predicted significant improvements in the surface finish when compared to CT. The model results in a $R a$ of a machined surface with a minimum accuracy of approximately $96 \%$ in the UVAT process.

The collected chips at $10 \mathrm{~m} / \mathrm{min}$ speed and the studied DoCs $(100 \mu \mathrm{m}, 200 \mu \mathrm{m}$ and $300 \mu \mathrm{m}$ ) resulted in a CCR of $0.64,0.72$, and 0.74 , respectively, while, the fuzzy model predicted a CCR $0.712,0.724$, and 0.749 , correspondingly. Additionally, at $20 \mathrm{~m} / \mathrm{min}$, the CCR predicted by the simulation were 0.75 and 0.79 , respectively, at $200 \mu \mathrm{m}$ and $300 \mu \mathrm{m}$ DoC. The experiments yielded 0.68 and 0.79 at the same cutting conditions. Likewise, at $30 \mathrm{~m} / \mathrm{min}$ and $300 \mu \mathrm{m} \mathrm{DoC}$, a minimal error of $0.5 \%$ and $0.25 \%$ was recorded between experimental and simulation results, respectively. The SA results predicted by the simulation model are in good agreement with the experimental results, and the model can be used to predict SA at the tested cutting conditions with an accuracy of $98.5 \%$ as presented in Table 7.

\section{Conclusions}

This paper reported the development of fuzzy-based simulation algorithms to predict the cutting forces, process zone temperature, surface quality of a machined specimen, CCR, and SA in the CT and UVAT processes. The algorithms were simulated effectively, and the results were in good agreement with the achieved experimental results. The simulation model for UVAT showed a significant reduction in the cutting forces and improvements in the surface quality when compared to the CT process. The developed model for the CT and UVAT processes can be used effectively to calculate the cutting forces, temperature of the process zone, surface quality, CCR, and SA of the studied alloys at various testing conditions within the acceptable range of accuracy (approximately 94\%). Additionally, these models can be helpful to avoid the extensive and costly experimental methods needed to estimate output machining parameters. These algorithms may require expert knowledge 
but can be good alternative for future industries and for the selection of cutting parameters in current industries.

Funding: This research received no external funding. The APC was funded by University of Bahrain, Kingdom of Bahrain.

Acknowledgments: The author would like to acknowledge the support of the Technical University of Braunschweig Germany, and Loughborough University, UK, in the current work.

Conflicts of Interest: The authors declare no conflict of interest.

\section{References}

1. Leyens, C.; Peters, M.; Hovsepian, P.; Lewis, D.; Luo, Q.; Münz, W.-D. Novel coating systems produced by the combined cathodic arc/unbalanced magnetron sputtering for environmental protection of titanium alloys. Surf. Coat. Technol. 2002, 155, 103-111. [CrossRef]

2. Arrazola, P.-J.; Garay, A.; Iriarte, L.-M.; Armendia, M.; Marya, S.; Le Maître, F. Machinability of titanium alloys (Ti6Al4V and Ti555. 3). J. Mater. Process. Technol. 2009, 209, 2223-2230. [CrossRef]

3. Maurotto, A.; Muhammad, R.; Roy, A.; Silberschmidt, V.V. Enhanced ultrasonically assisted turning of a $\beta$-titanium alloy. Ultrasonics 2013, 53, 1242-1250. [CrossRef]

4. Celaya, A.; de Lacalle, L.N.L.; Campa, F.J.; Lamikiz, A. Ultrasonic Assisted Turning of mild steels. Int. J. Mater. Prod. Technol. 2010, 37, 60. [CrossRef]

5. Celaya, A.; López de Lacalle, L.N.; Campa, F.J.; Lamikiz, A. Application of ultrasonics as assistance in machining operations. In Ultrasonics: Theory, Techniques and Practical Applications; Ayabito, H., Katsukawa, M., Eds.; Nova Science Publishers: New York, NY, USA, 2013; pp. 159-172.

6. Airao, J.; Nirala, C.; Lacalle, L.; Khanna, N. Tool Wear Analysis during Ultrasonic Assisted Turning of Nimonic-90 under Dry and Wet Conditions. Metals 2021, 11, 1253. [CrossRef]

7. Muhammad, R.; Ahmed, N.; Ullah, H.; Roy, A.; Silberschmidt, V.V. Hybrid machining process: Experimental and numerical analysis of hot ultrasonically assisted turning. Int. J. Adv. Manuf. Technol. 2018, 97, 2173-2192. [CrossRef]

8. Agrawal, C.; Wadhwa, J.; Pitroda, A.; Pruncu, C.I.; Sarikaya, M.; Khanna, N. Comprehensive analysis of tool wear, tool life, surface roughness, costing and carbon emissions in turning Ti-6Al-4V titanium alloy: Cryogenic versus wet machining. Tribol. Int. 2021, 153, 106597. [CrossRef]

9. Osman, K.A.; Yılmaz, V.; Ünver, H.Ö.; Şeker, U.; Kılıç, S.E. Slot milling of titanium alloy with hexagonal boron nitride and minimum quantity lubrication and multi-objective process optimization for energy efficiency. J. Clean. Prod. 2020, $258,120739$. [CrossRef]

10. Muhammad, R.; Hussain, M.S.; Maurotto, A.; Siemers, C.; Roy, A.; Silberschmidt, V.V. Analysis of a free machining $\alpha+\beta$ titanium alloy using conventional and ultrasonically assisted turning. J. Mater. Process. Technol. 2014, 214, 906-915. [CrossRef]

11. Suárez, A.; Veiga, F.; de Lacalle, L.N.L.; Polvorosa, R.; Lutze, S.; Wretland, A. Effects of Ultrasonics-Assisted Face Milling on Surface Integrity and Fatigue Life of Ni-Alloy 718. J. Mater. Eng. Perform. 2016, 25, 5076-5086. [CrossRef]

12. Parandoush, P.; Fernando, P.; Zhang, H.; Ye, C.; Xiao, J.; Zhang, M.; Lin, D. A finishing process via ultrasonic drilling for additively manufactured carbon fiber composites. Rapid Prototyp. J. 2021, 27, 754-768. [CrossRef]

13. Pujana, J.; Rivero, A.; Celaya, A.; de Lacalle, L.L. Analysis of ultrasonic-assisted drilling of Ti6Al4V. Int. J. Mach. Tools Manuf. 2009, 49, 500-508. [CrossRef]

14. Lotfi, M.; Akbari, J. Finite element simulation of ultrasonic-assisted machining: A review. Int. J. Adv. Manuf. Technol. 2021, 1-20. [CrossRef]

15. Lotfi, M.; Amini, S.; Akbari, J. Surface integrity and microstructure changes in 3D elliptical ultrasonic assisted turning of Ti-6Al-4V: FEM and experimental examination. Tribol. Int. 2020, 151, 106492. [CrossRef]

16. Pei, L.; Shu, X. Investigation of the turning process of the TC21 titanium alloy: Experimental analysis and 3D simulation. Proc. Inst. Mech. Eng. Part E J. Process. Mech. Eng. 2020, 0954408920967777. [CrossRef]

17. Muhammad, R.; Roy, A.; Silberschmidt, V.V. Finite Element Modelling of Conventional and Hybrid Oblique Turning Processes of Titanium Alloy. Procedia CIRP 2013, 8, 510-515. [CrossRef]

18. Muhammad, R. Hot Ultrasonically Assisted Turning of Ti-15V3Al3Cr3Sn: Experimental and Numerical Analysis; Loughborough University: Loughborough, UK, 2013.

19. Muhammad, R.; Ahmed, N.; Demiral, M.; Roy, A.; Silberschmidt, V.V. Computational Study of Ultrasonically-Assisted Turning of Ti Alloys. Adv. Mater. Res. 2011, 223, 30-36. [CrossRef]

20. Aamir, M.; Tu, S.; Tolouei-Rad, M.; Giasin, K.; Vafadar, A. Optimization and Modeling of Process Parameters in Multi-Hole Simultaneous Drilling Using Taguchi Method and Fuzzy Logic Approach. Materials 2020, 13, 680. [CrossRef]

21. Aamir, M.; Waqas, M.; Iqbal, M.; Hanif, M.I.; Muhammad, R. Fuzzy logic approach for investigation of microstructure and mechanical properties of Sn96.5-Ag3.0-Cu0.5 lead free solder alloy. Solder. Surf. Mt. Technol. 2017, 29, 191-198. [CrossRef]

22. Ubaid, A.M.; Dweiri, F.T.; Aghdeab, S.H.; Al-Juboori, L.A. Optimization of Electro Discharge Machining Process Parameters With Fuzzy Logic for Stainless Steel 304 (ASTM A240). J. Manuf. Sci. Eng. 2017, 140, 011013. [CrossRef] 
23. Hossain, A.; Hossain, A.; Nukman, Y.; Hassan, M.; Harizam, M.; Sifullah, A.; Parandoush, P.J.M. A fuzzy logic-based pre-diction model for kerf width in laser beam machining. Mater. Manuf. Process. 2016, 31, 679-684. [CrossRef]

24. Tseng, T.-L.; Konada, U.; Kwon, Y. A novel approach to predict surface roughness in machining operations using fuzzy set theory. J. Comput. Des. Eng. 2016, 3, 1-13. [CrossRef]

25. Senthilkumar, N.; Sudha, J.; Muthukumar, V. A grey-fuzzy approach for optimizing machining parameters and the approach angle in turning AISI 1045 steel. Adv. Prod. Eng. Manag. 2015, 10, 195-208. [CrossRef]

26. Ooi, M.E.; Sayuti, M.; Sarhan, A.A. Fuzzy logic-based approach to investigate the novel uses of nano suspended lubrication in precise machining of aerospace AL tempered grade 6061. J. Clean. Prod. 2015, 89, 286-295. [CrossRef]

27. Barzani, M.M.; Zalnezhad, E.; Sarhan, A.A.; Farahany, S.; Ramesh, S. Fuzzy logic based model for predicting surface roughness of machined Al-Si-Cu-Fe die casting alloy using different additives-turning. Measurement 2015, 61, 150-161. [CrossRef]

28. Vundavilli, P.R.; Parappagoudar, M.; Kodali, S.; Benguluri, S. Fuzzy logic-based expert system for prediction of depth of cut in abrasive water jet machining process. Knowl.-Based Syst. 2012, 27, 456-464. [CrossRef]

29. Ramesh, S.; Karunamoorthy, L.; Palanikumar, K. Fuzzy Modeling and Analysis of Machining Parameters in Machining Titanium Alloy. Mater. Manuf. Process. 2008, 23, 439-447. [CrossRef]

30. Kao, C.-C.; Shih, A.J.; Miller, S.F. Fuzzy Logic Control of Microhole Electrical Discharge Machining. J. Manuf. Sci. Eng. 2008, 130, 064502. [CrossRef]

31. Lin, C.-T.; Chung, I.-F.; Huang, S.-Y. Improvement of machining accuracy by fuzzy logic at corner parts for wire-EDM. Fuzzy Sets Syst. 2001, 122, 499-511. [CrossRef]

32. Lin, J.; Wang, K.; Yan, B.; Tarng, Y. Optimization of the electrical discharge machining process based on the Taguchi method with fuzzy logics. J. Mater. Process. Technol. 2000, 102, 48-55. [CrossRef]

33. Tzeng, Y.-F.; Chen, F.-C. Multi-objective optimisation of high-speed electrical discharge machining process using a Taguchi fuzzy-based approach. Mater. Des. 2007, 28, 1159-1168. [CrossRef]

34. Riaz, A.A.; Muhammad, R.; Ullah, N.; Hussain, G.; Alkahtani, M.; Akram, W. Fuzzy Logic-Based Prediction of Drill-ing-Induced Temperatures at Varying Cutting Conditions along with Analysis of Chips Morphology and Burrs Formation. Metals 2021, 11, 277. [CrossRef]

35. Malik, A.; Sanghvi, N. Optimization of laser-assisted jet electrochemical machining parameters by grey relational analysis and fuzzy logic. World J. Eng. 2021. [CrossRef]

36. Moganapriya, C.; Rajasekar, R.; Kumar, P.S.; Mohanraj, T.; Gobinath, V.; Saravanakumar, J. Achieving machining effec-tiveness for AISI 1015 structural steel through coated inserts and grey-fuzzy coupled Taguchi optimization approach. Struct. Multidiscip. Optim. 2021, 63, 1169-1186. [CrossRef]

37. Vignesh, M.; Ramanujam, R.; Rajyalakshmi, G.; Bhattacharya, S. Application of Grey Theory and Fuzzy Logic to Optimize Machining Parameters of Zircon Sand Reinforced Aluminum Composites. In Lecture Notes in Mechanical Engineering; Gabler: Lakewood, CO, USA, 2020; pp. 653-662.

38. Maurotto, A.; Siemers, C.; Muhammad, R.; Roy, A.; Silberschmidt, V.V. Ti Alloy with Enhanced Machinability in UAT Turning. Met. Mater. Trans. A 2014, 45, 2768-2775. [CrossRef]

39. Muhammad, R.; Maurotto, A.; Roy, A.; Silberschmidt, V.V. Ultrasonically assisted turning of Ti-6Al-2Sn-4Zr-6Mo. J. Phys. Conf. Ser. 2012, 382, 012016. [CrossRef]

40. Muhammad, R.; Maurotto, A.; Roy, A.; Silberschmidt, V.V. Analysis of Forces in Vibro-Impact and Hot Vibro-Impact Turning of Advanced Alloys. Appl. Mech. Mater. 2011, 70, 315-320. [CrossRef]

41. Pedrycz, W. Why triangular membership functions? Fuzzy Sets Syst. 1994, 64, 21-30. [CrossRef]

42. Ahmed, N.; Mitrofanov, A.V.; Babitsky, V.I.; Silberschmidt, V.V. Enhanced finite element model of ultrasonically assisted turning. Int. J. Mach. Mach. Mater. 2009, 6, 159. [CrossRef]

43. Ahmed, N.; Mitrofanov, A.; Babitsky, V.; Silberschmidt, V.V. 3D finite element analysis of ultrasonically assisted turning. Comput. Mater. Sci. 2007, 39, 149-154. [CrossRef]

44. Silberschmidt, V.V.; Mahdy, S.M.; Gouda, M.A.; Naseer, A.; Maurotto, A.; Roy, A. Surface-Roughness Improvement in UlTrasonically Assisted Turning. Procedia CIRP 2014, 13, 49-54. [CrossRef]

45. Thakur, D.G.; Ramamoorthy, B.; Vijayaraghavan, L. Machinability investigation of Inconel 718 in high-speed turning. Int. J. Adv. Manuf. Technol. 2009, 45, 421-429. [CrossRef]

46. Muhammad, R.; Ahmed, N.; Shariff, Y.M.; Silberschmidt, V.V. Effect of Cutting Conditions on Temperature Generated in Drilling Process: A FEA Approach. Adv. Mater. Res. 2011, 223, 240-246. [CrossRef]

47. Groover, M.P. Fundamentals of Modern Manufacturing: Materials, Processes, and Systems; John Wiley \& Sons: Hoboken, NJ, USA, 2020. 